\title{
DISSIPATIVE BOUNDARY CONDITIONS FOR ONE-DIMENSIONAL NONLINEAR HYPERBOLIC SYSTEMS*
}

\author{
JEAN-MICHEL CORON ${ }^{\dagger}$, GEORGES BASTIN ${ }^{\ddagger}$, AND BRIGITTE D’ANDRÉA-NOVEL ${ }^{\S}$
}

\begin{abstract}
We give a new sufficient condition on the boundary conditions for the exponential stability of one-dimensional nonlinear hyperbolic systems on a bounded interval. Our proof relies on the construction of an explicit strict Lyapunov function. We compare our sufficient condition with other known sufficient conditions for nonlinear and linear one-dimensional hyperbolic systems.
\end{abstract}

Key words. nonlinear hyperbolic systems, boundary conditions, stability, Lyapunov function

AMS subject classifications. 35F30, 35F25, 93D20, 93D30

DOI. $10.1137 / 070706847$

1. Introduction. We are concerned with the following one-dimensional $n \times n$ nonlinear hyperbolic system:

$$
u_{t}+F(u) u_{x}=0, \quad x \in[0,1], \quad t \in[0,+\infty),
$$

where $u:[0, \infty) \times[0,1] \rightarrow \mathbb{R}^{n}$ and $F: \mathbb{R}^{n} \rightarrow \mathcal{M}_{n, n}(\mathbb{R}), \mathcal{M}_{n, n}(\mathbb{R})$ denoting, as usual, the set of $n \times n$ real matrices. We consider the case where, possibly after an appropriate state transformation, $F(0)$ is a diagonal matrix with distinct and nonzero eigenvalues:

$$
\begin{gathered}
F(0):=\operatorname{diag}\left(\Lambda_{1}, \Lambda_{2}, \ldots, \Lambda_{n}\right), \\
\Lambda_{i}>0 \quad \forall i \in\{1, \ldots, m\}, \\
\Lambda_{i}<0 \quad \forall i \in\{m+1, \ldots, n\}, \\
\Lambda_{i} \neq \Lambda_{j} \quad \forall(i, j) \in\{1, \ldots, n\}^{2} \text { such that } i \neq j .
\end{gathered}
$$

In (1) and in what follows, diag $\left(\Lambda_{1}, \Lambda_{2}, \ldots, \Lambda_{n}\right)$ denotes the diagonal matrix whose $i$ th element on the diagonal is $\Lambda_{i}$.

Our concern is to analyze the asymptotic behavior of the classical solutions of the system under the following boundary condition:

$$
\left(\begin{array}{l}
u_{+}(t, 0) \\
u_{-}(t, 1)
\end{array}\right)=G\left(\begin{array}{l}
u_{+}(t, 1) \\
u_{-}(t, 0)
\end{array}\right), \quad t \in[0,+\infty),
$$

where the map $G: \mathbb{R}^{n} \rightarrow \mathbb{R}^{n}$ vanishes at 0 , while $u_{+} \in \mathbb{R}^{m}, u_{-} \in \mathbb{R}^{n-m}$ are defined by requiring that $u:=\left(u_{+}^{\mathrm{tr}}, u_{-}^{\mathrm{tr}}\right)^{\mathrm{tr}}$. The problem is to find the map $G$ such that

* Received by the editors October 30, 2007; accepted for publication (in revised form) January 16, 2008; published electronically May 7, 2008.

http://www.siam.org/journals/sicon/47-3/70684.html

${ }^{\dagger}$ Laboratoire Jacques-Louis Lions, Université Pierre et Marie Curie and Institut Universitaire de France, B.C. 187, 4 place Jussieu, 75252 Paris Cedex 05, France (coron@ann.jussieu.fr). This author's research was partially supported by the "Agence Nationale de la Recherche" (ANR), Project C-QUID, number BLAN-3-139579.

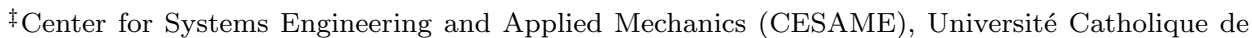
Louvain, 4 Avenue G. Lemaître, 1348 Louvain-la-Neuve, Belgium (Georges.Bastin@uclouvain.be).

${ }^{\S}$ Centre de Robotique, École des Mines de Paris, 60 boulevard Saint Michel, 75272 Paris Cedex 06, France (brigitte.dandrea-novel@ensmp.fr).

$$
1460
$$


the boundary condition (1.4) is dissipative, i.e., implies that the equilibrium solution $u \equiv 0$ of system (1.1) with the boundary condition (1.4) is exponentially stable.

This problem has been considered in the literature for more than 20 years. To our knowledge, the first results were published by Slemrod in [21] and Greenberg and $\mathrm{Li}$ in [9] for the special case of $2 \times 2$ (i.e., $u \in \mathbb{R}^{2}$ ) systems. A generalization to $n \times n$ systems was given by the Li school. Let us mention in particular [17] by Qin, [25] by Zhao, and [14, Theorem 1.3, page 173] by Li. All these results rely on a systematic use of direct estimates of the solutions and their derivatives along the characteristic curves. They give rise to sufficient dissipative boundary conditions which are kinds of "small gain conditions." The weakest sufficient condition [14, Theorem 1.3, page 173] is formulated as follows: $\rho\left(\left|G^{\prime}(0)\right|\right)<1$, where $\rho(A)$ denotes the spectral radius of $A \in \mathcal{M}_{n, n}(\mathbb{R})$ and $|A|$ denotes the matrix whose elements are the absolute values of the elements of $A \in \mathcal{M}_{n, n}(\mathbb{R})$.

In this paper we follow a different approach, which is based on a Lyapunov stability analysis. The special case of $2 \times 2$ systems and $F(u)$ diagonal has recently been treated in our previous paper [6]. In the present paper, by using a more general strict Lyapunov function (see section 4), we get a new weaker dissipative boundary condition, stated as follows:

$$
\operatorname{Inf}\left\{\left\|\Delta G^{\prime}(0) \Delta^{-1}\right\| ; \Delta \in \mathcal{D}_{n,+}\right\}<1,
$$

where \|\| denotes the usual 2-norm of matrices in $\mathcal{M}_{n, n}(\mathbb{R})$ and $\mathcal{D}_{n,+}$ denotes the set of diagonal matrices whose elements on the diagonal are strictly positive.

Moreover, our proof is rather elementary, and the existence of a strict Lyapunov function may be useful for studying robustness issues.

Our paper is organized as follows. In section 2, after some mathematical preliminaries, a precise technical definition of our new dissipative boundary condition is followed by the statement of our exponential stability theorem. Section 3 is then devoted to a discussion of the optimality properties of our dissipative boundary condition and to a comparison of this condition with other stability criteria from the literature, namely the criterion [14, Theorem 1.3, p, 173] mentioned above and a stability criterion for linear hyperbolic systems due to Silkowski. The proof of our exponential stability theorem, including the Lyapunov stability analysis, is thoroughly given in section 4. The paper ends with two appendices, where some technical properties of our dissipative boundary condition are given.

\section{A sufficient condition for exponential stability. For}

$$
x:=\left(x_{1}, \ldots, x_{n}\right)^{\operatorname{tr}} \in \mathbb{C}^{n},
$$

$|x|$ denotes the usual Hermitian norm of $x$ :

$$
|x|:=\sqrt{\sum_{i=1}^{n}\left|x_{i}\right|^{2}} .
$$

For $n \in \mathbb{N} \backslash\{0\}$ and $m \in \mathbb{N} \backslash\{0\}$, we denote by $\mathcal{M}_{n, m}(\mathbb{R})$ the set of $n \times m$ real matrices. We define, for $K \in \mathcal{M}_{n, m}(\mathbb{R})$,

$$
\|K\|:=\max \left\{|K x| ; x \in \mathbb{R}^{n},|x|=1\right\}=\max \left\{|K x| ; x \in \mathbb{C}^{n},|x|=1\right\},
$$

and, if $n=m$,

$$
\rho_{1}(K):=\operatorname{Inf}\left\{\left\|\Delta K \Delta^{-1}\right\| ; \Delta \in \mathcal{D}_{n,+}\right\}
$$


where $\mathcal{D}_{n,+}$ denotes the set of $n \times n$ real diagonal matrices with strictly positive diagonal elements.

For $\varepsilon$, let $B_{\varepsilon}$ be the open ball of $\mathbb{R}^{n}$ of radius $\varepsilon$. We assume that, for some $\varepsilon_{0}>0$, $F: B_{\varepsilon_{0}} \rightarrow \mathcal{M}_{n, n}(\mathbb{R})$ is of class $C^{2}$ and that there exists $m \in\{0, \ldots, n\}$ and $n$ real numbers $\Lambda_{1}, \ldots, \Lambda_{n}$ such that

$$
\begin{gathered}
\Lambda_{i}>0 \quad \forall i \in\{1, \ldots, m\} \text { and } \Lambda_{i}<0 \quad \forall i \in\{m+1, \ldots, n\}, \\
F(0)=\operatorname{diag}\left(\Lambda_{1}, \ldots, \Lambda_{n}\right), \\
\Lambda_{i} \neq \Lambda_{j} \quad \forall(i, j) \in\{1, \ldots, n\}^{2} \text { such that } i \neq j .
\end{gathered}
$$

For $u \in \mathbb{R}^{n}, u_{+} \in \mathbb{R}^{m}$ and $u_{-} \in \mathbb{R}^{n-m}$ are defined by requiring

$$
u=\left(\begin{array}{l}
u_{+} \\
u_{-}
\end{array}\right) .
$$

As mentioned in the introduction, we are mainly interested in analyzing the asymptotic convergence of the classical solutions of the following Cauchy problem:

$$
\begin{aligned}
& u_{t}+F(u) u_{x}=0, \quad x \in[0,1], \quad t \in[0,+\infty), \\
& \left(\begin{array}{l}
u_{+}(t, 0) \\
u_{-}(t, 1)
\end{array}\right)=G\left(\begin{array}{l}
u_{+}(t, 1) \\
u_{-}(t, 0)
\end{array}\right), \quad t \in[0,+\infty), \\
& u(0, x)=u^{0}(x), \quad x \in[0,1] .
\end{aligned}
$$

Concerning $G$, we assume that $G: B_{\varepsilon_{0}} \rightarrow \mathbb{R}^{n}$ is of class $C^{2}$ and satisfies $G(0)=0$. We define $F_{+}(u) \in \mathcal{M}_{m, n}(\mathbb{R}), F_{-}(u) \in \mathcal{M}_{(n-m), n}(\mathbb{R}), G_{+}(u) \in \mathbb{R}^{m}$, and $G_{-}(u) \in \mathbb{R}^{n-m}$ by requiring

$$
F(u)=\left(\begin{array}{l}
F_{+}(u) \\
F_{-}(u)
\end{array}\right), \quad G(u)=\left(\begin{array}{l}
G_{+}(u) \\
G_{-}(u)
\end{array}\right) .
$$

Regarding the existence of the solutions to the Cauchy problem (2.5)-(2.7), we have the following proposition.

Proposition 2.1. There exists $\delta_{0}>0$ such that, for every $u^{0} \in H^{2}\left((0,1), \mathbb{R}^{n}\right)$ satisfying

$$
\left|u^{0}\right|_{H^{2}\left((0,1), \mathbb{R}^{n}\right)} \leqslant \delta_{0}
$$

and the compatibility conditions

$$
\begin{gathered}
\left(\begin{array}{c}
u_{+}^{0}(0) \\
u_{-}^{0}(1)
\end{array}\right)=G\left(\begin{array}{c}
u_{+}^{0}(1) \\
u_{-}^{0}(0)
\end{array}\right), \\
F_{+}\left(u^{0}(0)\right) u_{x}^{0}(0)=\left[G_{+u_{+}}^{\prime}\left(\begin{array}{c}
u_{+}^{0}(1) \\
u_{-}^{0}(0)
\end{array}\right)\right] \\
F_{+}\left(u^{0}(1)\right) u_{x}^{0}(1) \\
+\left[G_{+u_{-}}^{\prime}\left(\begin{array}{c}
u_{+}^{0}(1) \\
u_{-}^{0}(0)
\end{array}\right)\right] F_{-}\left(u^{0}(0)\right) u_{x}^{0}(0),
\end{gathered}
$$

Copyright (C) by SIAM. Unauthorized reproduction of this article is prohibited. 


$$
\begin{aligned}
F_{-}\left(u^{0}(1)\right) u_{x}^{0}(1)=\left[G_{-u_{+}}^{\prime}\left(\begin{array}{c}
u_{+}^{0}(1) \\
u_{-}^{0}(0)
\end{array}\right)\right] & F_{+}\left(u^{0}(1)\right) u_{x}^{0}(1) \\
& +\left[G_{-u_{-}}^{\prime}\left(\begin{array}{c}
u_{+}^{0}(1) \\
u_{-}^{0}(0)
\end{array}\right)\right] F_{-}\left(u^{0}(0)\right) u_{x}^{0}(0),
\end{aligned}
$$

the Cauchy problem (2.5)-(2.7) has a unique maximal classical solution

$$
u \in C^{0}\left([0, T), H^{2}\left((0,1), \mathbb{R}^{n}\right)\right)
$$

with $T \in[0,+\infty]$. Moreover, if

$$
|u(t, \cdot)|_{H^{2}\left((0,1), \mathbb{R}^{n}\right)} \leqslant \delta_{0} \quad \forall t \in[0, T),
$$

then $T=+\infty$.

For a proof of this proposition, see, for instance, [12] by Kato, [13, pp. 2-3] by Lax, [16, pp. 35-43] by Majda, or [20, pp. 106-114] by Serre. Actually [12, 13, 16, 20] deal with $\mathbb{R}$ instead of $[0,1]$, but the proofs given there can be adapted to treat this new case. See also [15, pp. 96-107] by Li and Yu for the well-posedness of the Cauchy problem (2.5)-(2.7) in the framework of functions $u$ of class $C^{1}$. Let us briefly explain how to adapt these proofs in order to get, for example, the existence of a solution $u \in C^{0}\left([0, T], H^{2}\left((0,1), \mathbb{R}^{n}\right)\right)$ to the Cauchy problem (2.5)-(2.7) if $m=n$ (just to simplify the notation), for $T \in(0,+\infty)$ given, and for every $u^{0} \in H^{2}\left((0,1), \mathbb{R}^{n}\right)$ satisfying the compatibility conditions (2.8)-(2.9) (when $m=n$, condition (2.10) disappears) and such that $\left|u^{0}\right|_{H^{2}\left((0,1), \mathbb{R}^{n}\right)}$ is small enough (the smallness depending on $T$ in general). We first deal with the case where

$$
T \in\left(0, \min \left\{\Lambda_{1}^{-1}, \ldots, \Lambda_{n}^{-1}\right\}\right) .
$$

The basic ingredient is the following fixed point method, which is related to the one given in [15, page 97] (see also the pioneering works [12] and [13, pp. 2-3], where the authors deal with $\mathbb{R}$ instead of $[0,1])$. For $R>0$ and for $u^{0} \in H^{2}\left((0,1), \mathbb{R}^{n}\right)$ satisfying the compatibility conditions $(2.8)-(2.9)$, let $C_{R}\left(u^{0}\right)$ be the set of

$$
u \in L^{\infty}\left((0, T), H^{2}\left((0,1), \mathbb{R}^{n}\right)\right) \cap W^{1, \infty}\left((0, T), H^{1}\left((0,1), \mathbb{R}^{n}\right)\right)
$$

$$
\cap W^{2, \infty}\left((0, T), L^{2}\left((0,1), \mathbb{R}^{n}\right)\right)
$$

such that

$$
\begin{gathered}
|u|_{L^{\infty}\left((0, T), H^{2}\left((0,1), \mathbb{R}^{n}\right)\right)} \leqslant R, \\
|u|_{W^{1, \infty}\left((0, T), H^{1}\left((0,1), \mathbb{R}^{n}\right)\right)} \leqslant R, \\
|u|_{W^{2, \infty}\left((0, T), L^{2}\left((0,1), \mathbb{R}^{n}\right)\right)} \leqslant R, \\
u(\cdot, 1) \in H^{2}\left((0, T), \mathbb{R}^{n}\right) \text { and }|u(\cdot, 1)|_{H^{2}\left((0, T), \mathbb{R}^{n}\right)} \leqslant R^{2}, \\
u(0, \cdot)=u^{0}, \\
u_{t}(0, \cdot)=-F\left(u^{0}\right) u_{x}^{0} .
\end{gathered}
$$

Copyright $\odot$ by SIAM. Unauthorized reproduction of this article is prohibited. 
The set $C_{R}\left(u^{0}\right)$ is a closed subset of $L^{\infty}\left((0, T), L^{2}\left((0,1), \mathbb{R}^{n}\right)\right)$ (at least if $\left|u^{0}\right|_{H^{2}\left((0,1), \mathbb{R}^{n}\right)}$ is small enough so that $\left.\left|u^{0}\right|_{C^{0}\left([0,1], \mathbb{R}^{n}\right)}<\varepsilon_{0}\right)$. Given $R>0$, the set $C_{R}\left(u^{0}\right)$ is not empty if $\left|u^{0}\right|_{H^{2}\left((0,1), \mathbb{R}^{n}\right)}$ is small enough. Let $\mathcal{F}: C_{R}\left(u^{0}\right) \rightarrow L^{\infty}\left((0, T), H^{2}\left((0,1), \mathbb{R}^{n}\right)\right) \cap$ $W^{1, \infty}\left((0, T), H^{1}\left((0,1), \mathbb{R}^{n}\right)\right) \cap W^{2, \infty}\left((0, T), L^{2}\left((0,1), \mathbb{R}^{n}\right)\right)$ be defined by $\mathcal{F}(\tilde{u})=u$, where $u$ is the solution of the linear hyperbolic Cauchy problem

$$
\begin{gathered}
u_{t}+F(\tilde{u}) u_{x}=0, \quad u(t, 0)=G(\tilde{u}(t, 1)), \quad t \in[0, T], \\
u(0, x)=u^{0}(x), \quad x \in[0,1] .
\end{gathered}
$$

The set $C_{R}\left(u^{0}\right)$ is a closed subset of $L^{\infty}\left((0, T), L^{2}\left((0,1), \mathbb{R}^{n}\right)\right)$ (at least if $\left|u^{0}\right|_{H^{2}\left((0,1), \mathbb{R}^{n}\right)}$ is small enough so that $\left.\left|u^{0}\right|_{L^{\infty}\left((0,1), \mathbb{R}^{n}\right)} \leqslant \varepsilon_{0} / 2\right)$. Moreover, given $R>0, C_{R}\left(u^{0}\right)$ is not empty if $\left|u^{0}\right|_{H^{2}\left((0,1), \mathbb{R}^{n}\right)}$ is small enough. Using standard energy estimates and the finite speed of propagation inherent in (2.11), one gets the existence of $M>0$ and $R_{0}>0$ such that, for every $R \in\left(0, R_{0}\right]$, there exists $\delta>0$ such that, for every $u^{0} \in H^{2}\left((0,1), \mathbb{R}^{n}\right)$ such that $\left|u^{0}\right|_{H^{2}\left((0,1), \mathbb{R}^{n}\right)} \leqslant \delta$ and satisfying the compatibility conditions $(2.8)-(2.9)$,

$$
\mathcal{F}\left(C_{R}\left(u^{0}\right)\right) \subset C_{R}\left(u^{0}\right)
$$

and

$$
\begin{aligned}
& \left|\mathcal{F}\left(\tilde{u}_{2}\right)-\mathcal{F}\left(\tilde{u}_{1}\right)\right|_{L^{\infty}\left((0, T), L^{2}\left((0,1), \mathbb{R}^{n}\right)\right)}+M\left|\mathcal{F}\left(\tilde{u}_{2}\right)(\cdot, 1)-\mathcal{F}\left(\tilde{u}_{1}\right)(\cdot, 1)\right|_{L^{2}\left((0,1), \mathbb{R}^{n}\right)} \\
\leqslant & \frac{1}{2}\left|\tilde{u}_{2}-\tilde{u}_{1}\right|_{L^{\infty}\left((0, T), L^{2}\left((0,1), \mathbb{R}^{n}\right)\right)}+\frac{M}{2}\left|\tilde{u}_{2}(\cdot, 1)-\tilde{u}_{1}(\cdot, 1)\right|_{L^{2}\left((0,1), \mathbb{R}^{n}\right)} \quad \forall\left(\tilde{u}_{1}, \tilde{u}_{2}\right) \in C_{R}\left(u^{0}\right) .
\end{aligned}
$$

This allows us to prove that $\mathcal{F}$ has a fixed point $u \in C_{R}\left(u^{0}\right)$; i.e., there exists a solution $u \in C_{R}\left(u^{0}\right)$ to the Cauchy problem (2.5)-(2.7). In order to get the extra regularity property $u \in C^{0}\left([0, T], H^{2}\left((0,1), \mathbb{R}^{n}\right)\right)$, one can adapt $[16$, pp. $44-46]$ by noticing that, when one uses usual energy estimates to get $(2.12)$, one also gets, for $u:=\mathcal{F}(\tilde{u})$ with $\tilde{u} \in C_{R}\left(u^{0}\right)$, the "hidden regularity" $u_{x x}(\cdot, 1) \in L^{2}\left((0, T), \mathbb{R}^{n}\right)$ together with estimates on $\left|u_{x x}(\cdot, 1)\right|_{L^{2}\left((0, T), \mathbb{R}^{n}\right)}$ which are sufficient to take care of the boundary terms which now appear when one does integrations by parts. The case of general $T \in(0+\infty)$ follows by applying the above result to $\left[0, T_{1}\right],\left[T_{1}, 2 T_{1}\right],\left[2 T_{1}, 3 T_{1}\right], \ldots$, with $T_{1}$ given in $\left(0, \min \left\{\Lambda_{1}^{-1}, \ldots, \Lambda_{n}^{-1}\right\}\right)$. This concludes our sketch of the proof of Proposition 2.1.

We adopt the following definition of the exponential stability of the equilibrium solution $u \equiv 0$.

DeFINITION 2.2. The equilibrium solution $u \equiv 0$ of the nonlinear hyperbolic system (2.5)-(2.6) is exponentially stable (for the $H^{2}$-norm) if there exist $\varepsilon>0, \nu>0$, and $C>0$ such that, for every $u^{0} \in H^{2}\left((0,1), \mathbb{R}^{n}\right)$ satisfying $\left|u^{0}\right|_{H^{2}\left((0,1), \mathbb{R}^{n}\right)} \leqslant \varepsilon$ and the compatibility conditions (2.8)-(2.10), the classical solution $u$ to the Cauchy problem $(2.5)-(2.7)$ is defined on $[0,+\infty)$ and satisfies

$$
|u(t, \cdot)|_{H^{2}\left((0,1), \mathbb{R}^{n}\right)} \leqslant C e^{-\nu t}\left|u_{0}\right|_{H^{2}\left((0,1), \mathbb{R}^{n}\right)} \quad \forall t \in[0,+\infty) .
$$

Our main result is the following theorem.

THEOREM 2.3. If $\rho_{1}\left(G^{\prime}(0)\right)<1$, then the equilibrium $u \equiv 0$ of the quasi-linear hyperbolic system $(2.5)-(2.6)$ is exponentially stable.

The proof of this theorem is given in section 4 .

As mentioned in the introduction, the next section is devoted to a comparison of our dissipative boundary condition (i.e., $\rho_{1}\left(G^{\prime}(0)\right)<1$ ) with other stability criteria from the literature, namely the criterion given in [14, Theorem 1.3, page 173] and a stability criterion for linear hyperbolic systems. 
3. Comparison with other stability conditions. In this section, we first compare our condition $\rho_{1}\left(G^{\prime}(0)\right)<1$ for exponential stability (Theorem 2.3) with a prior condition found by $\mathrm{Li}[14$, Theorem 1.3, page 173]. In the second part of this section we shall compare our condition to conditions for the stability of linear hyperbolic systems.

3.1. Comparison with the Li condition. Let us first introduce some notation and definitions. For $K \in \mathcal{M}_{n, m}(\mathbb{R})$, we denote by $K_{i j}$ the term on the $i$ th line and $j$ th column of the matrix $K$ and denote by $|K|$ the matrix in $\mathcal{M}_{n, m}(\mathbb{R})$ defined by

$$
|K|_{i j}:=\left|K_{i j}\right| \quad \forall i \in\{1, \ldots, n\}, \forall j \in\{1, \ldots, m\} .
$$

We define, for $K \in \mathcal{M}_{n, n}(\mathbb{R})$,

$$
\begin{gathered}
R_{2}(K):=\operatorname{Max}\left\{\sum_{j=1}^{n}\left|K_{i j}\right| ; i \in\{1, \ldots, n\}\right\}, \\
\rho_{2}(K):=\operatorname{Inf}\left\{R_{2}\left(\Delta K \Delta^{-1}\right) ; \Delta \in \mathcal{D}_{n,+}\right\} .
\end{gathered}
$$

Note that, by [14, Lemma 2.4, page 146],

$$
\rho_{2}(K)=\rho(|K|),
$$

where, for $A \in \mathcal{M}_{n, n}(\mathbb{R}), \rho(A)$ is the spectral radius of $A$. In the following theorem, we recall the sufficient condition for exponential stability introduced by Li.

Theorem 3.1 (see [14, Theorem 1.3, page 173]). Assume that $\rho_{2}\left(G^{\prime}(0)\right)<$ 1 ; then $0 \in C^{1}\left([0,1], \mathbb{R}^{n}\right)$ is locally exponentially stable in the $C^{1}([0,1])$-norm for the hyperbolic system (2.5)-(2.6); i.e., there exist $\varepsilon>0, \nu>0$, and $C>0$ such that, for every $u^{0} \in C^{1}\left([0,1], \mathbb{R}^{n}\right)$ satisfying $\left|u^{0}\right|_{C^{1}\left([0,1], \mathbb{R}^{n}\right)} \leqslant \varepsilon$ and the compatibility conditions (2.8)-(2.10), the Cauchy problem (2.5)-(2.7) has a unique solution $u$ in $C^{1}\left([0,+\infty) \times[0,1], \mathbb{R}^{n}\right)$, and this solution satisfies

$$
|u(t, \cdot)|_{C^{1}\left([0,1], \mathbb{R}^{n}\right)} \leqslant C e^{-\nu t}\left|u^{0}\right|_{C^{1}\left([0,1], \mathbb{R}^{n}\right)} \quad \forall t \in[0,+\infty) .
$$

The following proposition and (3.3) show that our new sufficient condition, namely $\rho_{1}\left(G^{\prime}(0)\right)<1$, is weaker than the previous one.

Proposition 3.2. For every $K \in \mathcal{M}_{n, n}(\mathbb{R})$,

$$
\rho_{1}(K) \leqslant \rho_{2}(K) \text {. }
$$

Let us point out that there are matrices $K$ such that inequality (3.2) is strict. For example, for $a>0$, let

$$
K_{a}:=\left(\begin{array}{cc}
a & a \\
-a & a
\end{array}\right) \in \mathcal{M}_{2,2}(\mathbb{R}) .
$$

Then

$$
\rho_{1}\left(K_{a}\right)=\sqrt{2} a<2 a=\rho_{2}\left(K_{a}\right) .
$$

Remark 3.3. In fact, in [14, Theorem 1.3, page 173], it is assumed that $G_{+}$ depends only on $u_{-}$and that $G_{-}$depends only on $u_{+}$. However, if one takes

$$
K:=\left(\begin{array}{cc}
0 & K_{a} \\
K_{a} & 0
\end{array}\right) \in \mathcal{M}_{4,4}(\mathbb{R}),
$$

Copyright @ by SIAM. Unauthorized reproduction of this article is prohibited. 
$n=4, m=2$, and $G(u):=K u$, which are allowed by the type of boundary conditions considered in [14, Theorem 1.3, page 173], one again gets $\rho_{1}(K)=\sqrt{2} a<2 a=$ $\rho_{2}(K)$.

Proof of Proposition 3.2. Let us first prove the following lemma.

Lemma 3.4. For every $K \in \mathcal{M}_{n, n}(\mathbb{R})$, for every $D \in \mathcal{D}_{n,+}$, for every $\Delta \in \mathcal{D}_{n,+}$, for every $X \in \mathbb{R}^{n}$, and for every $Y \in \mathbb{R}^{n}$,

$$
Y^{\operatorname{tr}} \Delta K \Delta^{-1} X \leqslant \frac{1}{2} R_{2}\left(D \Delta^{-1} K^{\operatorname{tr}} \Delta D^{-1}\right)|X|^{2}+\frac{1}{2} R_{2}\left(D \Delta K \Delta^{-1} D^{-1}\right)|Y|^{2} .
$$

Proof of Lemma 3.4. Replacing, if necessary, $K$ by $\Delta K \Delta^{-1}$, we may assume without loss of generality that $\Delta$ is the identity map of $\mathbb{R}^{n}$. We write $X:=\left(X_{1}, \ldots, X_{n}\right)^{\operatorname{tr}} \in$ $\mathbb{R}^{n}, Y:=\left(Y_{1}, \ldots, Y_{n}\right)^{\operatorname{tr}} \in \mathbb{R}^{n}, D:=\operatorname{diag}\left(D_{1}, \ldots, D_{n}\right)$. One has

$$
\begin{aligned}
Y^{\mathrm{tr}} K X & =\sum_{i=1}^{n} Y_{i}\left(\sum_{j=1}^{n} K_{i j} X_{j}\right)=\sum_{i=1}^{n} \sum_{j=1}^{n} \frac{K_{i j}}{D_{i} D_{j}} D_{i} Y_{i} D_{j} X_{j} \\
& \leqslant \frac{1}{2} Q_{1}+\frac{1}{2} Q_{2},
\end{aligned}
$$

with

$$
Q_{1}:=\sum_{i=1}^{n} \sum_{j=1}^{n} \frac{\left|K_{i j}\right|}{D_{i} D_{j}} D_{j}^{2} X_{j}^{2} \text { and } Q_{2}:=\sum_{i=1}^{n} \sum_{j=1}^{n} \frac{\left|K_{i j}\right|}{D_{i} D_{j}} D_{i}^{2} Y_{i}^{2} .
$$

Note that

$$
\begin{aligned}
Q_{1} & \left.=\sum_{j=1}^{n}\left(\sum_{i=1}^{n}\left|K_{i j}\right| D_{i}^{-1} D_{j}\right) X_{j}^{2}=\sum_{j=1}^{n}\left(\sum_{i=1}^{n} \mid\left(D^{-1} K D\right)^{\operatorname{tr}}\right)_{j i} \mid\right) X_{j}^{2} \\
& \leqslant \sum_{j=1}^{n} R_{2}\left(\left(D^{-1} K D\right)^{\operatorname{tr}}\right) X_{j}^{2}=R_{2}\left(D K^{\operatorname{tr}} D^{-1}\right)|X|^{2} .
\end{aligned}
$$

Similarly,

$$
\begin{aligned}
Q_{2} & =\sum_{i=1}^{n}\left(\sum_{j=1}^{n} D_{i}\left|K_{i j}\right| D_{j}^{-1}\right) Y_{i}^{2}=\sum_{i=1}^{n}\left(\sum_{j=1}^{n}\left|\left(D K D^{-1}\right)_{i j}\right|\right) Y_{i}^{2} \\
& \leqslant \sum_{i=1}^{n} R_{2}\left(D K D^{-1}\right) Y_{i}^{2}=R_{2}\left(D K D^{-1}\right)|Y|^{2} .
\end{aligned}
$$

Inequality (3.4) follows from (3.5), (3.6), and (3.7). This concludes the proof of Lemma 3.4.

Let us go back to the proof of Proposition 3.2. One easily sees that

$$
\left\{\left(D \Delta^{-1}, D \Delta\right) ; D \in \mathcal{D}_{n,+}, \Delta \in \mathcal{D}_{n,+}\right\}=\mathcal{D}_{n,+} \times \mathcal{D}_{n,+} .
$$

Equality (3.8) implies that

$$
\begin{aligned}
& \rho_{2}\left(K^{\operatorname{tr}}\right)+\rho_{2}(K) \\
& =\operatorname{Inf}\left\{R_{2}\left(D \Delta^{-1} K^{\operatorname{tr}} \Delta D^{-1}\right)+R_{2}\left(D \Delta K \Delta^{-1} D^{-1}\right) ; D \in \mathcal{D}_{n,+}, \Delta \in \mathcal{D}_{n,+}\right\} .
\end{aligned}
$$

Copyright (? by SIAM. Unauthorized reproduction of this article is prohibited. 
Using (3.1), we have

$$
\rho_{2}\left(K^{\mathrm{tr}}\right)=\rho\left(\left|K^{\mathrm{tr}}\right|\right)=\rho\left(|K|^{\mathrm{tr}}\right)=\rho(|K|)=\rho_{2}(K),
$$

which, together with (3.9), gives

$$
\begin{array}{r}
\operatorname{Inf}\left\{R_{2}\left(D \Delta^{-1}|K|^{\operatorname{tr}} \Delta D^{-1}\right)+R_{2}\left(D \Delta|K| \Delta^{-1} D^{-1}\right) ; D \in \mathcal{D}_{n,+}, \Delta \in \mathcal{D}_{n,+}\right\} \\
=2 \rho_{2}(K) .
\end{array}
$$

Finally, let us note that, for every $\Delta$ in $\mathcal{D}_{n,+}$,

$$
\operatorname{Sup}\left\{Y^{\operatorname{tr}} \Delta K \Delta^{-1} X ; X \in \mathbb{R}^{n}, Y \in \mathbb{R}^{n},|X|=|Y|=1\right\}=\left\|\Delta K \Delta^{-1}\right\| \geqslant \rho_{1}(K) .
$$

Proposition 3.2 follows from (3.4), (3.11), and (3.12).

3.2. Comparison with stability conditions for linear hyperbolic systems. Replacing, if necessary, $y(t, x)$ by

$$
\left(\begin{array}{c}
y_{+}(t, x) \\
y_{-}(t, 1-x)
\end{array}\right),
$$

it may be assumed, without loss of generality, that the speeds of propagation $\Lambda_{i}$ are all positive. More precisely we consider the special case of linear hyperbolic systems

$$
y_{t}+\Lambda y_{x}=0, \quad y(t, 0)=K y(t, 1)
$$

where

$$
\Lambda:=\operatorname{diag}\left(\Lambda_{1}, \ldots, \Lambda_{n}\right), \quad \text { with } \Lambda_{i}>0 \quad \forall i \in\{1, \ldots, n\} .
$$

In order to avoid compatibility conditions, one can deal with the case where $y(t, \cdot) \in$ $L^{2}\left((0,1), \mathbb{R}^{n}\right)$ (instead of $y(t, \cdot) \in H^{2}\left((0,1), \mathbb{R}^{n}\right)$, as we consider above for the nonlinear hyperbolic system (2.5)-(2.6)). It is well known that the Cauchy problem associated with (3.13) is well posed in $L^{2}\left((0,1), \mathbb{R}^{n}\right)$; that is, for every $y^{0} \in L^{2}\left((0,1), \mathbb{R}^{n}\right)$, there exists a unique

$$
y \in C^{0}\left([0,+\infty), L^{2}\left((0,1), \mathbb{R}^{n}\right)\right)
$$

solution of (3.13) satisfying the initial condition

$$
y(0, \cdot)=y^{0} .
$$

Of course, (3.13) has to be understood in the classical weak sense; i.e., for every $\varphi \in C^{1}\left([0,+\infty) \times[0,1] ; \mathbb{R}^{n}\right)$ with compact support and satisfying

$$
\varphi^{\operatorname{tr}}(t, 1) \Lambda-\varphi_{+}^{\operatorname{tr}}(t, 0) \Lambda K=0 \quad \forall t \in[0,+\infty),
$$

we have

$$
\int_{0}^{+\infty} \int_{0}^{1}\left(\varphi_{t}^{\operatorname{tr}}+\varphi_{x}^{\operatorname{tr}} \Lambda\right) y d x d t+\int_{0}^{1} \varphi^{\operatorname{tr}}(0, x) y^{0}(x) d x=0
$$

Copyright (C) by SIAM. Unauthorized reproduction of this article is prohibited. 
See, for example, [5, section 2.1].

As usual, we say that $0 \in L^{2}\left((0,1), \mathbb{R}^{n}\right)$ is exponentially stable for (3.13) (for the norm of $\left.L^{2}\left((0,1), \mathbb{R}^{n}\right)\right)$ if there exist $\nu>0$ and $C>0$ such that, for every $y^{0} \in L^{2}\left((0,1), \mathbb{R}^{n}\right)$, the solution of the Cauchy problem (3.13), (3.15) satisfies

$$
|y(t, \cdot)|_{L^{2}\left((0,1), \mathbb{R}^{n}\right)} \leqslant C e^{-\nu t}\left|y^{0}\right|_{L^{2}\left((0,1), \mathbb{R}^{n}\right)} \quad \forall t \in[0,+\infty) .
$$

One easily checks that (3.13) is equivalent to

$$
\phi_{i}(t)=\sum_{j=1}^{n} K_{i j} \phi_{j}\left(t-r_{j}\right) \quad \forall i \in\{1, \ldots, n\},
$$

with

$$
\phi_{j}(t):=y_{j}(t, 0), \quad r_{j}:=\frac{1}{\Lambda_{j}}, \quad j \in\{1, \ldots, n\} .
$$

Hence (3.13) can be considered as a linear time-delay system. By a classical result on linear time-delay systems (see, e.g., [10, Theorem 3.5 page 275] by Hale and Verduyn Lunel), $0 \in L^{2}\left((0,1), \mathbb{R}^{n}\right)$ is exponentially stable for the system (3.13) if and only if there exists $\delta>0$ such that

$$
\left(\operatorname{det}\left(\operatorname{Id}_{n}-\left(\operatorname{diag}\left(e^{-r_{1} z}, \ldots, e^{-r_{n} z}\right)\right) K\right)=0, z \in \mathbb{C}\right) \Rightarrow(\Re(z) \leqslant-\delta),
$$

where $\operatorname{Id}_{n}$ is the identity map of $\mathbb{R}^{n}$ and $\Re(z)$ denotes the real part of the complex number $z$. Note that $\rho_{1}(K)<1$ implies the existence of $\delta>0$ such that (3.17) holds. Indeed, let us assume that $\rho_{1}(K)<1$. Then, by $(2.1)$, there exist $\mu \in(0,1)$ and $D \in \mathcal{D}_{n,+}$ such that

$$
\left\|D K D^{-1}\right\| \leqslant \mu .
$$

Let us assume that $z \in \mathbb{C}$ is such that

$$
\operatorname{det}\left(\operatorname{Id}_{n}-\left(\operatorname{diag}\left(e^{-r_{1} z}, \ldots, e^{-r_{n} z}\right)\right) K\right)=0 .
$$

Then

$$
\begin{aligned}
& \operatorname{det}\left(\operatorname{Id}_{n}-\left(\operatorname{diag}\left(e^{-r_{1} z}, \ldots, e^{-r_{n} z}\right)\right) D K D^{-1}\right) \\
&=\operatorname{det}\left(D\left(\operatorname{Id}_{n}-\left(\operatorname{diag}\left(e^{-r_{1} z}, \ldots, e^{-r_{n} z}\right)\right) K\right) D^{-1}\right) \\
&=\operatorname{det}\left(\operatorname{Id}_{n}-\left(\operatorname{diag}\left(e^{-r_{1} z}, \ldots, e^{-r_{n} z}\right)\right) K\right)=0,
\end{aligned}
$$

which implies that

$$
\left.\|\left(\operatorname{diag}\left(e^{-r_{1} z}, \ldots, e^{-r_{n} z}\right)\right) D K D^{-1}\right) \| \geqslant 1 .
$$

Since

$$
\begin{aligned}
\left.\|\left(\operatorname{diag}\left(e^{-r_{1} z}, \ldots, e^{-r_{n} z}\right)\right) D K D^{-1}\right) \| & \leqslant\left\|\operatorname{diag}\left(e^{-r_{1} z}, \ldots, e^{-r_{n} z}\right)\right\|\left\|D K D^{-1}\right\| \\
& \leqslant e^{-\min \left\{r_{1} \Re(z), \ldots, r_{n} \Re(z)\right\}}\left\|D K D^{-1}\right\|,
\end{aligned}
$$

Copyright $@$ by SIAM. Unauthorized reproduction of this article is prohibited. 
one has, also using (3.18) and (3.19),

$$
e^{-\min \left\{r_{1} \Re(z), \ldots, r_{n} \Re(z)\right\}} \mu \geqslant 1 .
$$

Inequality (3.20) implies that (3.17) holds with $\delta:=\ln (\mu) /\left(\max \left\{r_{1}, \ldots, r_{n}\right\}\right)<0$.

The converse is false: the existence of $\delta>0$ such that (3.17) holds does not imply that $\rho_{1}(K)<1$. For example, let us choose $r_{1}:=1, r_{2}:=2$, and

$$
K:=\left(\begin{array}{ll}
a & a \\
a & a
\end{array}\right), \quad a \in \mathbb{R} .
$$

(This example is borrowed from [10, page 285].) It is easily seen that $\rho_{1}(K)=2|a|$. Hence $\rho_{1}(K)<1$ is equivalent to $a \in(-1 / 2,1 / 2)$. However, the existence of $\delta>0$ such that (3.17) holds is equivalent to $a \in(-1,1 / 2)$.

If we want to try to apply results on the stability of the linear hyperbolic system (3.13) in order to get the stability of our nonlinear hyperbolic system (2.5)-(2.6), since $F(u)$ depends on $u$, it is natural to ask for the robustness of the stability of the linear hyperbolic system (3.13) with respect to small changes on the $\Lambda_{i}$ 's, i.e., on the speeds of propagation. (One can easily sees that the stability is robust with respect to small changes on $K$.) Let us adopt the following definition.

DEFINITION 3.5. The linear system (3.13) is robustly exponentially stable with respect to the speeds of propagation if there exists $\varepsilon>0$ such that, for every $\tilde{\Lambda}:=$ $\operatorname{diag}\left(\tilde{\Lambda}_{1}, \ldots, \tilde{\Lambda}_{n}\right) \in \mathcal{D}_{n,+}$ such that

$$
\left|\tilde{\Lambda}_{i}-\Lambda_{i}\right| \leqslant \varepsilon \quad \forall i \in\{1, \ldots, n\},
$$

$0 \in L^{2}\left((0,1), \mathbb{R}^{n}\right)$ is exponentially stable for the perturbed linear hyperbolic system

$$
y_{t}+\tilde{\Lambda} y_{x}=0, \quad y(t, 0)=K y(t, 1)
$$

One has, then, the following theorem, which is due to Silkowski (see [10, Theorem 6.1 , page 286]; see also $[26,11])$.

TheOREM 3.6. Let

$$
\rho_{0}(K):=\max \left\{\rho\left(\operatorname{diag}\left(e^{\iota \theta_{1}}, \ldots, e^{\iota \theta_{n}}\right) K\right) ;\left(\theta_{1}, \ldots, \theta_{n}\right)^{\operatorname{tr}} \in \mathbb{R}^{n}\right\},
$$

with $\iota:=\sqrt{-1}$. If the $\left(r_{1}, \ldots, r_{n}\right)$ are rationally independent, the linear system (3.13) is exponentially stable if and only if $\rho_{0}(K)<1$. In particular (note that $\rho_{0}(K)$ depends continuously on $K)$, whatever $\left(r_{1}, \ldots, r_{n}\right) \in(0,+\infty)^{n}$ is, the linear system (3.13) is robustly exponentially stable with respect to the speeds of propagation if and only if $\rho_{0}(K)<1$.

From this theorem the interest of comparing $\rho_{0}(K)$ and $\rho_{1}(K)$ is clear. This is done in the following proposition.

Proposition 3.7. For every $n \in \mathbb{N}$ and for every $K \in \mathcal{M}_{n, n}(\mathbb{R})$,

$$
\rho_{0}(K) \leqslant \rho_{1}(K) .
$$

For every $n \in\{1,2,3,4,5\}$ and for every $K \in \mathcal{M}_{n, n}(\mathbb{R})$,

$$
\rho_{0}(K)=\rho_{1}(K) \text {. }
$$

For every $n \in \mathbb{N} \backslash\{1,2,3,4,5\}$, there exists $K \in \mathcal{M}_{n, n}(\mathbb{R})$ such that

$$
\rho_{0}(K)<\rho_{1}(K) \text {. }
$$

The proof of Proposition 3.7 is given in Appendix B. 
4. Proof of Theorem 2.3. For the clarity of the analysis, we first deal in detail with the case where $m=n$ and then give only the main modifications to deal with the case $m<n$. When $m=n$ the boundary condition (2.6) reads

$$
u(t, 0)=G(u(t, 1)), \quad t \in[0,+\infty),
$$

and the compatibility conditions (2.8)-(2.10) become

$$
\begin{gathered}
u^{0}(0)=G\left(u^{0}(1)\right), \\
F\left(u^{0}(0)\right) u_{x}^{0}(0)=G^{\prime}\left(u^{0}(1)\right) F\left(u^{0}(1)\right) u_{x}^{0}(1) .
\end{gathered}
$$

Let us introduce some simplifying notation,

$$
\Lambda:=F(0) \in \mathcal{D}_{n,+}, \quad K:=G^{\prime}(0), \quad v:=u_{x}, \quad w:=v_{x}=u_{x x},
$$

and let us denote by $\mathcal{S}_{n}$ the set of $n \times n$ real symmetric matrices and by $\mathcal{S}_{n,+}$ the set of $n \times n$ real symmetric positive definite matrices.

We shall repeatedly use the following lemma.

Lemma 4.1. Let $\Lambda:=\operatorname{diag}\left(\Lambda_{1}, \ldots, \Lambda_{n}\right) \in \mathcal{D}_{n}$ be such that (2.4) holds. Let $\Delta \in$ $\mathcal{D}_{n}$. Then there exist a positive real number $\eta$ and a map $N:\left\{M \in \mathcal{M}_{n, n}(\mathbb{R}) ; \| M-\right.$ $\Lambda \|<\eta\} \rightarrow \mathcal{S}_{n}$ of class $C^{\infty}$ such that

$$
\begin{gathered}
N(\Lambda)=\Delta, \\
N(M) M-M^{t r} N(M)=0 \quad \forall M \in \mathcal{M}_{n, n}(\mathbb{R}) \text { such that }\|M-\Lambda\|<\eta .
\end{gathered}
$$

Proof of Lemma 4.1. Let $\mathcal{A}_{n}$ be the set of matrices $A \in \mathcal{M}_{n, n}(\mathbb{R})$ such that $A^{\text {tr }}=-A$. For $M \in \mathcal{M}_{n, n}(\mathbb{R})$, let us consider the following linear map:

$$
\begin{aligned}
\mathcal{L}_{M}: \mathcal{S}_{n} & \rightarrow & \mathcal{A}_{n} \times \mathcal{D}_{n}, \\
S & \mapsto & \left(S M-M^{\operatorname{tr}} S, \operatorname{Diag}(S)\right),
\end{aligned}
$$

where $\operatorname{Diag}(S):=\operatorname{diag}\left(S_{11}, \ldots, S_{n n}\right)$. Noticing that

$$
S \Lambda-\Lambda^{\operatorname{tr}} S=\left(\Lambda_{j}-\Lambda_{i}\right) S_{i j} \quad \forall i \in\{1, \ldots, n\}, \forall j \in\{1, \ldots, n\}, \forall S \in \mathcal{S}_{n}
$$

and using (2.4), it is easily checked that $\mathcal{L}_{\Lambda}: \mathcal{S}_{n} \rightarrow \mathcal{A}_{n} \times \mathcal{D}_{n}$ is an isomorphism. Hence there exists $\eta>0$ such that, for every $M \in \mathcal{M}_{n, n}(\mathbb{R})$ such that $\|M-\Lambda\|<\eta$, $\mathcal{L}_{M}$ is an isomorphism. It then suffices to define $N$ by

$$
N(M)=\mathcal{L}_{M}^{-1}(0, \Delta) .
$$

This concludes the proof of Lemma 4.1.

For the stability analysis, we now introduce the Lyapunov function candidate

$$
V(u, v, w)=V_{1}(u)+V_{2}(u, v)+V_{3}(u, v, w),
$$

with

$$
\begin{aligned}
& V_{1}(u)=\int_{0}^{1} u^{\operatorname{tr}} Q(u) u e^{-\mu x} d x \\
& V_{2}(u, v)=\int_{0}^{1} v^{\operatorname{tr}} R(u) v e^{-\mu x} d x \\
& V_{3}(u, v, w)=\int_{0}^{1} w^{\operatorname{tr}} S(u) w e^{-\mu x} d x
\end{aligned}
$$

Copyright $@$ by SIAM. Unauthorized reproduction of this article is prohibited. 
where $\mu>0, Q(u), R(u)$, and $S(u)$ are symmetric positive definite matrices which will be defined later.

Remark 4.2. The weight $e^{-\mu x}$ is essential to get a strict Lyapunov function. It is similar to the one introduced in [4] to stabilize the Euler equation of incompressible fluids (see the definition of $V$ given on page 1886 of [4]). It has also been used by $\mathrm{Xu}$ and Sallet in [24] for quite general linear hyperbolic systems (see also [22] by Tchousso, Besson, and $\mathrm{Xu}$ ).

Let us compute the time derivative $\dot{V}_{1}$ of $V_{1}$ along the classical $C^{1}$-solutions of system (2.5) with boundary conditions (4.1). One has

$$
\begin{aligned}
\dot{V}_{1} & =\int_{0}^{1}\left\{2 u^{\operatorname{tr}} Q(u) u_{t}+u^{\operatorname{tr}}(Q(u))_{t} u\right\} e^{-\mu x} d x \\
& =\int_{0}^{1}\left\{-2 u^{\operatorname{tr}} Q(u) F(u) u_{x}+u^{\operatorname{tr}}\left[Q^{\prime}(u) u_{t}\right] u\right\} e^{-\mu x} d x,
\end{aligned}
$$

where $Q^{\prime}(u)$ is the linear map from $\mathbb{R}^{n}$ to $\mathcal{S}_{n}$ which stands for the derivative of $Q$ at the point $u$. Hence

$$
\dot{V}_{1}=\int_{0}^{1}\left\{-\left(u^{\operatorname{tr}} Q(u) F(u) u\right)_{x}+u^{\operatorname{tr}}(Q(u) F(u))_{x} u-u^{\operatorname{tr}}\left[Q^{\prime}(u) F(u) v\right] u\right\} e^{-\mu x} d x .
$$

For $f \in C^{0}\left([0,1], \mathbb{R}^{n}\right)$, we denote by $|f|_{0}$ its $C^{0}$-norm: $|f|_{0}:=\max \{|f(x)| ; x \in[0,1]\}$. From now on, $V_{1}$ and $\dot{V}_{1}$ are considered as functionals defined, respectively, by (4.6) and (4.9) on the set $\mathcal{V}_{1}$ of $u \in C^{1}\left([0,1], \mathbb{R}^{n}\right)$ satisfying $|u|_{0}<\varepsilon_{0}$ and the compatibility condition

$$
u_{0}=G\left(u_{1}\right) \text {, }
$$

with $u_{0}:=u(0)$ and $u_{1}:=u(1)$.

Since $\rho_{1}(K)<1$ by assumption, there exists $D \in \mathcal{D}_{n,+}$ such that $\left\|D K D^{-1}\right\|<1$. The matrix $Q(u)$ is selected as the matrix $N(F(u))$ of Lemma 4.1 with $\Delta:=D^{2} \Lambda^{-1}$.

Our estimates on $V_{1}$ and $\dot{V}_{1}$ are in the following lemma.

Lemma 4.3. There exists $\mu_{1}>0$ such that, for every $\mu \in\left(0, \mu_{1}\right)$, there exist positive real constants $\alpha_{1}, \beta_{1}, \delta_{1}$ such that, for every $u \in \mathcal{V}_{1}$ such that $|u|_{0} \leqslant \delta_{1}$,

$$
\begin{aligned}
& \frac{1}{\beta_{1}} \int_{0}^{1}|u|^{2} d x \leqslant V_{1}(u) \leqslant \beta_{1} \int_{0}^{1}|u|^{2} d x, \\
& \dot{V}_{1}(u) \leqslant-\alpha_{1} V_{1}(u)+\beta_{1} \int_{0}^{1}|u|^{2}\left|u_{x}\right| d x .
\end{aligned}
$$

Proof of Lemma 4.3. Throughout this proof, $u$ is assumed to be in $\mathcal{V}_{1}$. From the construction of $Q$,

$$
Q(0) F(0)=Q(0) \Lambda=D^{2} \in \mathcal{D}_{n,+},
$$

and there exists $\delta_{11} \in\left(0, \varepsilon_{0} / 2\right)$ such that

$$
Q(a) \in \mathcal{S}_{n,+} \text { and } Q(a) F(a) \in \mathcal{S}_{n,+} \quad \forall a \in \mathbb{R}^{n} \text { such that }|a| \leqslant \delta_{11} .
$$

Copyright $@$ ㅇ by SIAM. Unauthorized reproduction of this article is prohibited. 
Clearly, from (4.14), we obtain that, for every $\mu>0$, there exists $\beta_{1}>0$ such that (4.11) holds if $|u|_{0} \leqslant \delta_{11}$.

Let us now deal with the estimate (4.12) on $\dot{V}_{1}\left(=\dot{V}_{1}(u)\right)$. Let us decompose $\dot{V}_{1}$ in the following way:

$$
\dot{V}_{1}=\mathcal{T}_{11}+\mathcal{T}_{12}+\mathcal{T}_{13}
$$

with

$$
\begin{aligned}
& \mathcal{T}_{11}:=-\mu \int_{0}^{1}\left(u^{\operatorname{tr}} Q(u) F(u) u\right) e^{-\mu x} d x, \\
& \mathcal{T}_{12}:=-\int_{0}^{1}\left(u^{\operatorname{tr}} Q(u) F(u) u e^{-\mu x}\right)_{x} d x, \\
& \mathcal{T}_{13}:=\int_{0}^{1}\left\{u^{\operatorname{tr}}\left(\left[Q^{\prime}(u) v\right] F(u)+Q(u)\left[F^{\prime}(u) v\right]-\left[Q^{\prime}(u) F(u) v\right]\right) u\right\} e^{-\mu x} d x .
\end{aligned}
$$

Analysis of the first term $\mathcal{T}_{11}$. By (4.11) and (4.14), for every $\mu>0$, there exists a positive real constant $\alpha_{1}>0$ such that, if $|u|_{0} \leqslant \delta_{11}$,

$$
\mathcal{T}_{11} \leqslant-\alpha_{1} V_{1}
$$

Analysis of the second term $\mathcal{T}_{12}$. One has

$$
\begin{aligned}
\mathcal{T}_{12} & =-\left[u^{\operatorname{tr}} Q(u) F(u) u e^{-\mu x}\right]_{0}^{1} \\
& =-\left(u_{1}^{\operatorname{tr}} Q\left(u_{1}\right) F\left(u_{1}\right) u_{1} e^{-\mu}-u_{0}^{\operatorname{tr}} Q\left(u_{0}\right) F\left(u_{0}\right) u_{0}\right) .
\end{aligned}
$$

Let us introduce a notation in order to deal with estimates on "higher order terms." We denote by $\mathcal{O}(X, Y)$, with $X \geqslant 0$ and $Y \geqslant 0$, quantities such that there exist $C>0$ and $\varepsilon>0$, independent of $u, v$ and $w$, satisfying

$$
(Y \leqslant \varepsilon) \Rightarrow(|\mathcal{O}(X, Y)| \leqslant C X) .
$$

Using the compatibility condition (4.10), we have

$$
\begin{aligned}
\mathcal{T}_{12} & =-\left(u_{1}^{\operatorname{tr}} Q\left(u_{1}\right) F\left(u_{1}\right) u_{1} e^{-\mu}-\left(G\left(u_{1}\right)\right)^{\operatorname{tr}} Q\left(G\left(u_{1}\right)\right) F\left(G\left(u_{1}\right)\right) G\left(u_{1}\right)\right) \\
& =-u_{1}^{\operatorname{tr}}\left(Q(0) \Lambda e^{-\mu}-K^{\operatorname{tr}} Q(0) \Lambda K\right) u_{1}+\mathcal{O}\left(\left|u_{1}\right|^{3} ;\left|u_{1}\right|\right) .
\end{aligned}
$$

For $u_{1} \in \mathbb{R}^{n}$, we define $\zeta:=D u_{1}$. Then, using (4.13), we have, for every $u_{1} \in \mathbb{R}^{n}$,

$$
u_{1}^{\operatorname{tr}} K^{\operatorname{tr}} Q(0) \Lambda K u_{1}=u_{1}^{\operatorname{tr}} K^{\operatorname{tr}} D D K u_{1}=\left(\zeta^{\operatorname{tr}} D^{-1} K^{\operatorname{tr}} D\right)\left(D K D^{-1} \zeta\right)=\left|D K D^{-1} \zeta\right|^{2} .
$$

Hence, using (4.13) once again, we have, for every $u_{1} \in \mathbb{R}^{n}$,

$$
u_{1}^{\operatorname{tr}} K^{\operatorname{tr}} Q(0) \Lambda K u_{1} \leqslant\left\|D K D^{-1}\right\|^{2} \zeta^{\operatorname{tr}} \zeta=\left\|D K D^{-1}\right\|^{2} u_{1}^{\operatorname{tr}} Q(0) \Lambda u_{1} .
$$

From this inequality and the fact that $\left\|D K D^{-1}\right\|<1$, it follows that, taking $\mu>0$ small enough (which is always implicitly assumed), $Q(0) \Lambda e^{-\mu}-K^{\operatorname{tr}} Q(0) \Lambda K$ is a 
positive definite matrix. Then, using (4.20), there exists $\delta_{12}>0$ such that, if $\left|u_{1}\right| \leqslant$ $\delta_{12}$,

$$
\mathcal{T}_{12} \leqslant 0 .
$$

Analysis of the third term $\mathcal{T}_{13}$. The integrand of $\mathcal{T}_{13}$ is linear with respect to $v$ and, at least, quadratic with respect to $u$. It follows that, increasing the value of $\beta_{1}$ if necessary, there exists a real positive constant $\delta_{13}$ such that, for $|u|_{0} \leqslant \delta_{13}$,

$$
\mathcal{T}_{13} \leqslant \beta_{1} \int_{0}^{1}|u|^{2}|v| d x .
$$

Then, collecting inequalities (4.19), (4.22), and (4.23) together, if

$$
|u|_{0} \leqslant \delta_{1}:=\min \left\{\delta_{11}, \delta_{12}, \delta_{13}\right\}
$$

we conclude that

$$
\dot{V}_{1}=\mathcal{T}_{11}+\mathcal{T}_{12}+\mathcal{T}_{13} \leqslant-\alpha_{1} V_{1}+\beta_{1} \int_{0}^{1}|u|^{2}|v| d x .
$$

This completes the proof of Lemma 4.3.

From Lemma 4.3 it appears that it is clearly necessary to examine the dynamics of $v=u_{x}$ in order to carry out the Lyapunov stability analysis. This is the reason why the Lyapunov function (4.5) is extended with terms involving $v$. By time differentiation of the system equations (2.5) and (4.1), it may be shown that $v$ satisfies the dynamics

$$
\begin{aligned}
& v_{t}+F(u) v_{x}+\left[F^{\prime}(u) v\right] v=0, \quad x \in[0,1], \quad t \in[0,+\infty), \\
& F(u(t, 0)) v(t, 0)=G^{\prime}(u(t, 1)) F(u(t, 1)) v(t, 1), \quad t \in[0,+\infty) .
\end{aligned}
$$

Let us compute the time derivative of $V_{2}$ along the classical $C^{1}$-solutions of system (4.25) with boundary conditions (4.26). One has

$$
\begin{aligned}
\dot{V}_{2} & =\int_{0}^{1}\left\{2 v^{\operatorname{tr}} R(u) v_{t}+v^{\operatorname{tr}}(R(u))_{t} v\right\} e^{-\mu x} d x \\
& =\int_{0}^{1}\left\{-2 v^{\operatorname{tr}} R(u) F(u) v_{x}-2 v^{\operatorname{tr}} R(u)\left[F^{\prime}(u) v\right] v-v^{\operatorname{tr}}\left[R^{\prime}(u) F(u) v\right] v\right\} e^{-\mu x} d x .
\end{aligned}
$$

From now on, $V_{2}$ and $\dot{V}_{2}$ are considered as functionals defined, respectively, by (4.7) and (4.27) on the set $\mathcal{V}_{2}$ of $(u, v) \in C^{2}\left([0,1], \mathbb{R}^{n}\right) \times C^{1}\left([0,1], \mathbb{R}^{n}\right)$ such that

$$
\begin{gathered}
|u|_{0}<\varepsilon_{0}, \\
u_{x}=v, \\
u_{0}=G\left(u_{1}\right), \\
F\left(u_{0}\right) v_{0}=G^{\prime}\left(u_{1}\right) F\left(u_{1}\right) v_{1},
\end{gathered}
$$

where $u_{0}:=u(0), u_{1}:=u(1)$ as above, and $v_{0}:=v(0), v_{1}:=v(1)$. 
The matrix $R(u)$ is selected as the matrix $N(F(u))$ of Lemma 4.1 now with $\Delta:=\Lambda D^{2}$. Our estimates on $V_{2}$ and $\dot{V}_{2}$ are in the following lemma.

Lemma 4.4. There exists $\mu_{2}>0$ such that, for every $\mu \in\left(0, \mu_{2}\right)$, there exist positive real constants $\alpha_{2}, \beta_{2}, \delta_{2}$ such that, for every $(u, v) \in \mathcal{V}_{2}$ such that $|u|_{0} \leqslant \delta_{2}$,

$$
\begin{gathered}
\frac{1}{\beta_{2}} \int_{0}^{1}|v|^{2} d x \leqslant V_{2}(u, v) \leqslant \beta_{2} \int_{0}^{1}|v|^{2} d x, \\
\dot{V}_{2}(u, v) \leqslant-\alpha_{2}\left(V_{2}(u, v)+\left|v_{1}\right|^{2}\right)+\beta_{2} \int_{0}^{1}|v|^{3} d x .
\end{gathered}
$$

Proof of Lemma 4.4. Throughout this proof, $(u, v)$ is assumed to be in $\mathcal{V}_{2}$. By the construction of $R$, we have

$$
F(0)^{-1} R(0)=\Lambda^{-1} R(0)=D^{2} \in \mathcal{D}_{n,+}
$$

and the existence of $\delta_{21} \in\left(0, \varepsilon_{0} / 2\right)$ such that

$$
R(a) \in \mathcal{S}_{n,+} \text { and } R(a) F(a) \in \mathcal{S}_{n,+} \quad \forall a \in \mathbb{R}^{n} \text { such that }|a| \leqslant \delta_{21} .
$$

Clearly, from (4.35), for every $\mu>0$, there exists $\beta_{2}>0$ such that (4.32) holds if $|u|_{0} \leqslant \delta_{21}$.

Let us now deal with the estimate (4.33) on $\dot{V}_{2}\left(=\dot{V}_{2}(u, v)\right)$. Let us decompose $\dot{V}_{2}$ in the following way:

$$
\dot{V}_{2}=\mathcal{T}_{21}+\mathcal{T}_{22}+\mathcal{T}_{23}
$$

with

$$
\begin{aligned}
& \mathcal{T}_{21}:=-\mu \int_{0}^{1}\left(v^{\operatorname{tr}} R(u) F(u) v\right) e^{-\mu x} d x \\
& \mathcal{T}_{22}:=-\int_{0}^{1}\left(v^{\operatorname{tr}} R(u) F(u) v e^{-\mu x}\right)_{x} d x \\
& \mathcal{T}_{23}:=\int_{0}^{1}\left\{v^{\operatorname{tr}}\left(\left[(R(u) F(u))_{x} v\right]-2 R(u)\left[F^{\prime}(u) v\right]-\left[R^{\prime}(u) F(u) v\right]\right) v\right\} e^{-\mu x} d x
\end{aligned}
$$

Analysis of the first term $\mathcal{T}_{21}$. By (4.32) and (4.35), for every $\mu>0$, there exists a positive real constant $\alpha_{21}>0$ such that, if $|u|_{0} \leqslant \delta_{21}$,

$$
\mathcal{T}_{21} \leqslant-\alpha_{21} V_{2} .
$$

Analysis of the second term $\mathcal{T}_{22}$. One has

$$
\begin{aligned}
\mathcal{T}_{22} & =-\left[v^{\operatorname{tr}} R(u) F(u) v e^{-\mu x}\right]_{0}^{1} \\
& =-\left(v_{1}^{\operatorname{tr}} R\left(u_{1}\right) F\left(u_{1}\right) v_{1} e^{-\mu}-v_{0}^{\operatorname{tr}} R\left(u_{0}\right) F\left(u_{0}\right) v_{0}\right) .
\end{aligned}
$$

Under the boundary condition (4.31), we have

$$
\begin{aligned}
\mathcal{T}_{22}=-v_{1}^{\operatorname{tr}}\left(R\left(u_{1}\right) F\left(u_{1}\right) e^{-\mu}-F\left(u_{1}\right)^{\operatorname{tr}}\left(G^{\prime}\left(u_{1}\right)\right)^{\operatorname{tr}}\right. & \\
& \left.\left(F\left(G\left(u_{1}\right)\right)^{-1}\right)^{\operatorname{tr}} R\left(G\left(u_{1}\right)\right) G^{\prime}\left(u_{1}\right) F\left(u_{1}\right)\right) v_{1},
\end{aligned}
$$

Copyright $(\odot$ by SIAM. Unauthorized reproduction of this article is prohibited. 
which implies that

$$
\mathcal{T}_{22}=-v_{1}^{\operatorname{tr}}\left(R(0) \Lambda e^{-\mu}-\Lambda K^{\operatorname{tr}} \Lambda^{-1} R(0) K \Lambda\right) v_{1}+\mathcal{O}\left(\left|v_{1}\right|^{2}\left|u_{1}\right| ;\left|u_{1}\right|\right) .
$$

We define $\zeta:=D v_{1}$. Then, using (4.34), we have, for every $v_{1} \in \mathbb{R}^{n}$,

$$
v_{1}^{\mathrm{tr}} K^{\mathrm{tr}} \Lambda^{-1} R(0) K v_{1}=v_{1}^{\mathrm{tr}} K^{\mathrm{tr}} D D K v_{1}=\left(\zeta^{\mathrm{tr}} D^{-1} K^{\operatorname{tr}} D\right)\left(D K D^{-1} \zeta\right)=\left|D K D^{-1} \zeta\right|^{2} .
$$

Therefore, using (4.34) once again, we get that, for every $v_{1} \in \mathbb{R}^{n}$,

$$
v_{1}^{\operatorname{tr}} K^{\operatorname{tr}} \Lambda^{-1} R(0) K v_{1} \leqslant\left\|D K D^{-1}\right\|^{2} \zeta^{\operatorname{tr}} \zeta=\left\|D K D^{-1}\right\|^{2} v_{1}^{\operatorname{tr}} \Lambda^{-1} R(0) v_{1} .
$$

From (4.40) and the fact that $\left\|D K D^{-1}\right\|<1$, it follows that, choosing $\mu>0$ small enough, $\Lambda^{-1} R(0) e^{-\mu}-K^{\operatorname{tr}} \Lambda^{-1} R(0) K$ is a positive definite matrix, which, in turn, implies that the matrix $R(0) \Lambda e^{-\mu}-\Lambda K^{\operatorname{tr}} \Lambda^{-1} R(0) K \Lambda$ is also positive definite. Hence there exist $\alpha_{22}>0$ and $\delta_{22}>0$ such that, if $\left|u_{1}\right| \leqslant \delta_{22}$, we have

$$
\mathcal{T}_{22} \leqslant-\alpha_{22}\left|v_{1}\right|^{2}
$$

Analysis of the third term $\mathcal{T}_{23}$. The integrand of $\mathcal{T}_{23}$ is, at least, cubic with respect to $v$. It follows that, increasing the value of $\beta_{2}$ if necessary, there exists $\delta_{23} \in\left(0, \varepsilon_{0} / 2\right)$ such that, for $|u|_{0} \leqslant \delta_{23}$,

$$
\mathcal{T}_{23} \leqslant \beta_{2} \int_{0}^{1}|v|^{3} d x .
$$

Then, collecting inequalities (4.37), (4.41), and (4.42) together, we conclude that if $|u|_{0} \leqslant \delta_{2}:=\min \left\{\delta_{21}, \delta_{22}, \delta_{23}\right\}$ and if $\alpha_{2}:=\min \left\{\alpha_{21}, \alpha_{22}\right\}$, then

$$
\dot{V}_{2}=\mathcal{T}_{21}+\mathcal{T}_{22}+\mathcal{T}_{23} \leqslant-\alpha_{2}\left(V_{2}+\left|v_{1}\right|^{2}\right)+\beta_{2} \int_{0}^{1}|v|^{3} d x .
$$

This completes the proof of Lemma 4.4 .

Note that $V_{2}$ is not sufficient to get an upper bound on $\int_{0}^{1}|v|^{3} d x$. For that reason, we also need to consider the dynamics of $w$ to complete the Lyapunov stability analysis. By a further time differentiation of the system equations (4.25)-(4.26), we obtain

$$
\begin{array}{r}
w_{t}+F(u) w_{x}+\left[F^{\prime}(u) w\right] v+2\left[F^{\prime}(u) v\right] w+\left[F^{\prime \prime}(u)(v, v)\right] v=0, \\
x \in[0,1], \quad t \in[0,+\infty),
\end{array}
$$

under the boundary condition

$$
F\left(u_{0}\right) w_{0}+\left[F^{\prime}\left(u_{0}\right) v_{0}\right] v_{0}=\left[H^{\prime}\left(u_{1}\right) F\left(u_{1}\right) v_{1}\right] v_{1}+H\left(u_{1}\right) F\left(u_{1}\right) w_{1}+H\left(u_{1}\right)\left[F^{\prime}\left(u_{1}\right) v_{1}\right] v_{1},
$$

with the notation $w_{0}:=w(0), w_{1}:=w(1)$, and $H(u):=F(G(u))^{-1} G^{\prime}(u) F(u)$. Using the previous boundary conditions (4.30) and (4.31), this boundary condition (4.45) may be written in compact form as

$$
w_{0}=F\left(G\left(u_{1}\right)\right)^{-1} H\left(u_{1}\right) F\left(u_{1}\right) w_{1}+\mathcal{Z}\left(u_{1}, v_{1}\right),
$$


where $\mathcal{Z}$ is continuous on a neighborhood of $0 \in \mathbb{R}^{n} \times \mathbb{R}^{n}$ and such that

$$
\mathcal{Z}\left(u_{1}, v_{1}\right)=\mathcal{O}\left(\left|v_{1}\right|^{2} ;\left|u_{1}\right|\right) \text {. }
$$

Let us compute the time derivative of $V_{3}$ along the classical $C^{1}$-solutions of system (4.44) with boundary conditions (4.46). One has

$$
\begin{gathered}
\dot{V}_{3}=\int_{0}^{1}\left\{2 w^{\operatorname{tr}} S(u) w_{t}+w^{\operatorname{tr}}(S(u))_{t} w\right\} e^{-\mu x} d x \\
=\int_{0}^{1}\left\{-2 w^{\operatorname{tr}} S(u) F(u) w_{x}-2 w^{\operatorname{tr}} S(u)\left(\left[F^{\prime}(u) w\right] v+2\left[F^{\prime}(u) v\right] w+\left[F^{\prime \prime}(u)(v, v)\right] v\right)\right. \\
\left.-w^{\operatorname{tr}}\left[S^{\prime}(u) F(u) v\right] w\right\} e^{-\mu x} d x .
\end{gathered}
$$

From now on, $V_{3}$ and $\dot{V}_{3}$ are considered as functionals defined, respectively, by (4.8) and (4.48) on the set $\mathcal{V}_{3}$ of $(u, v, w) \in C^{3}\left([0,1], \mathbb{R}^{n}\right) \times C^{2}\left([0,1], \mathbb{R}^{n}\right) \times C^{1}\left([0,1], \mathbb{R}^{n}\right)$ such that

$$
\begin{gathered}
|u|_{0}<\varepsilon_{0}, \\
u_{x}=v, \quad v_{x}=w, \\
u_{0}=G\left(u_{1}\right), \\
F\left(u_{0}\right) v_{0}=G^{\prime}\left(u_{1}\right) F\left(u_{1}\right) v_{1}, \\
w_{0}=F\left(G\left(u_{1}\right)\right)^{-1} H\left(u_{1}\right) F\left(u_{1}\right) w_{1}+\mathcal{Z}\left(u_{1}, v_{1}\right),
\end{gathered}
$$

with $u_{0}:=u(0), u_{1}:=u(1), v_{0}:=v(0)$, and $v_{1}:=v(1)$ as above, and $w_{0}:=w(0)$ and $w_{1}:=w(1)$.

The matrix $S(u)$ is selected as the matrix $N(F(u))$ of Lemma 4.1 now with $\Delta:=\Lambda^{2} D^{2} \Lambda$. Our estimates on $V_{3}$ and $\dot{V}_{3}$ are in the following lemma.

Lemma 4.5. There exists $\mu_{3}>0$ such that, for every $\mu \in\left(0, \mu_{3}\right)$, there exist positive real constants $\alpha_{3}, \beta_{3}, \delta_{3}$ such that, for every $(u, v, w) \in \mathcal{V}_{3}$ such that $|u|_{0}+$ $|v|_{0} \leqslant \delta_{3}$, one has

$$
\begin{gathered}
\frac{1}{\beta_{3}} \int_{0}^{1}|w|^{2} d x \leqslant V_{3}(u, v, w) \leqslant \beta_{3} \int_{0}^{1}|w|^{2} d x \\
\dot{V}_{3}(u, v, w) \leqslant-\alpha_{3} V_{3}(u, v, w)+\beta_{3}\left|v_{1}\right|^{4}+\beta_{3} \int_{0}^{1}\left(|v|^{2}|w|+|w|^{2}|v|\right) d x .
\end{gathered}
$$

Proof of Lemma 4.5. Throughout this proof, we assume that $(u, v, w) \in \mathcal{V}_{3}$. By the construction of $S$, we have

$$
\Lambda^{-2} S(0) \Lambda^{-1}=D^{2} \in \mathcal{D}_{n,+}
$$

and the existence of $\delta_{31} \in\left(0, \varepsilon_{0} / 2\right)$ such that

$$
S(a) \in \mathcal{S}_{n,+} \text { and } S(a) F(a) \in \mathcal{S}_{n,+} \quad \forall a \in \mathbb{R}^{n} \text { such that }|u| \leqslant \delta_{31} .
$$

Clearly, from (4.57), we obtain that, for every $\mu>0$, there exists $\beta_{3}>0$ such that (4.54) holds if $|u|_{0} \leqslant \delta_{31}$.

Copyright (? by SIAM. Unauthorized reproduction of this article is prohibited. 
Let us now deal with the estimate (4.55) on $\dot{V}_{3}\left(=\dot{V}_{3}(u, v, w)\right)$. Let us decompose $\dot{V}_{3}$ in the following way:

$$
\dot{V}_{3}=\mathcal{T}_{31}+\mathcal{T}_{32}+\mathcal{T}_{33}
$$

with

$$
\begin{gathered}
\mathcal{T}_{31}:=-\mu \int_{0}^{1}\left(w^{\operatorname{tr}} S(u) F(u) w\right) e^{-\mu x} d x \\
\mathcal{T}_{32}:=-\int_{0}^{1}\left(w^{\operatorname{tr}} S(u) F(u) w e^{-\mu x}\right)_{x} d x \\
\mathcal{T}_{33}:=-\int_{0}^{1}\left\{-w^{\operatorname{tr}}\left(\left[(S(u) F(u))_{x} v\right]+\left[S^{\prime}(u) F(u) v\right]\right) w+2 w^{\operatorname{tr}} S(u)\left[F^{\prime}(u) w\right] v\right. \\
\left.+4 w^{\operatorname{tr}} S(u)\left[F^{\prime}(u) v\right] w+2 w^{\operatorname{tr}}\left[F^{\prime \prime}(u)(v, v)\right] v\right\} e^{-\mu x} d x .
\end{gathered}
$$

Analysis of the first term $\mathcal{T}_{31}$. By (4.54) and (4.57), for every $\mu>0$, there exists a positive real constant $\alpha_{3}>0$ such that, if $|u|_{0} \leqslant \delta_{31}$,

$$
\mathcal{T}_{31} \leqslant-\alpha_{3} V_{3}
$$

Analysis of the second term $\mathcal{T}_{32}$.

$$
\begin{aligned}
\mathcal{T}_{32} & =-\left[w^{\operatorname{tr}} S(u) F(u) w e^{-\mu x}\right]_{0}^{1} \\
& =-\left(w_{1}^{\operatorname{tr}} S\left(u_{1}\right) F\left(u_{1}\right) w_{1} e^{-\mu}-w_{0}^{\operatorname{tr}} S\left(u_{0}\right) F\left(u_{0}\right) w_{0}\right) .
\end{aligned}
$$

Under the boundary conditions (4.51) and (4.53), we have, also using (4.47),

$$
\begin{aligned}
& \mathcal{T}_{32}=-w_{1}^{\operatorname{tr}}\left(S\left(u_{1}\right) F\left(u_{1}\right) e^{-\mu}\right. \\
&\left.-F\left(u_{1}\right)^{\operatorname{tr}} H\left(u_{1}\right)^{\operatorname{tr}}\left(F\left(G\left(u_{1}\right)\right)^{-1}\right)^{\operatorname{tr}} S\left(G\left(u_{1}\right)\right) H\left(u_{1}\right) F\left(u_{1}\right)\right) w_{1} \\
&+ \\
&+\mathcal{O}\left(\left|v_{1}\right|^{4}+\left|v_{1}\right|^{2}\left|w_{1}\right| ;\left|u_{1}\right|\right) \\
&=-w_{1}^{\operatorname{tr}}\left(S(0) \Lambda e^{-\mu}-\Lambda^{2} K^{\operatorname{tr}} \Lambda^{-2}\right.\left.S(0) \Lambda^{-1} K \Lambda^{2}\right) w_{1} \\
&+\mathcal{O}\left(\left|v_{1}\right|^{4}+\left|v_{1}\right|^{2}\left|w_{1}\right|+\left|w_{1}\right|^{2}\left|u_{1}\right| ;\left|u_{1}\right|\right) .
\end{aligned}
$$

For $w_{1} \in \mathbb{R}^{n}$, we define $\zeta:=D w_{1}$. Then, using (4.56), we have, for every $w_{1} \in \mathbb{R}^{n}$,

$$
\begin{aligned}
w_{1}^{\operatorname{tr}} K^{\operatorname{tr}} \Lambda^{-2} S(0) \Lambda^{-1} K w_{1} & =w_{1}^{\operatorname{tr}} K^{\operatorname{tr}} D D K w_{1} \\
& =\left(\zeta^{\operatorname{tr}} D^{-1} K^{\operatorname{tr}} D\right)\left(D K D^{-1} \zeta\right)=\left|D K D^{-1} \zeta\right|^{2} .
\end{aligned}
$$

Therefore, for every $w_{1} \in \mathbb{R}^{n}$, we have, using (4.56) once again,

$$
w_{1}^{\mathrm{tr}} K^{\operatorname{tr}} \Lambda^{-2} S(0) \Lambda^{-1} K w_{1} \leqslant\left\|D K D^{-1}\right\|^{2} \zeta^{\operatorname{tr}} \zeta=\left\|D K D^{-1}\right\|^{2} w_{1}^{\operatorname{tr}} \Lambda^{-2} S(0) \Lambda^{-1} w_{1} .
$$

Copyright (? by SIAM. Unauthorized reproduction of this article is prohibited. 
From this inequality and the fact that $\left\|D K D^{-1}\right\|^{2}<1$, it follows that, choosing $\mu>0$ small enough, $\Lambda^{-2} S(0) \Lambda^{-1} e^{-\mu}-K^{\operatorname{tr}} \Lambda^{-2} S(0) \Lambda^{-1} K$ is a positive definite symmetric matrix, which, in turn, implies that the matrix

$$
S(0) \Lambda e^{-\mu}-\Lambda^{2} K^{\operatorname{tr}} \Lambda^{-2} S(0) \Lambda^{-1} K \Lambda^{2}
$$

is also positive definite. Moreover, for every $\eta>0$ and for every $\left(v_{1}, w_{1}\right) \in \mathbb{R}^{n} \times \mathbb{R}^{n}$,

$$
\left|v_{1}\right|^{2}\left|w_{1}\right| \leqslant \frac{1}{4 \eta}\left|v_{1}\right|^{4}+\eta\left|w_{1}\right|^{2} .
$$

Hence, taking $\eta>0$ small enough and also using (4.60), one gets the existence of $\delta_{32}>0$ and $\beta_{32}>0$ such that, if $|u|_{0}+|v|_{0} \leqslant \delta_{32}$,

$$
\mathcal{T}_{32} \leqslant \beta_{32}\left|v_{1}\right|^{4} .
$$

Analysis of the third term $\mathcal{T}_{33}$. Note that

$$
(F(u) G(u))_{x}=\left[F^{\prime}(u) v\right] G(u)+F(u) G^{\prime}(u) v .
$$

It follows that there exist $\delta_{33}>0$ and $\beta_{33}>0$ such that, if $|u|_{0}+|v|_{0} \leqslant \delta_{33}$, then

$$
\mathcal{T}_{33} \leqslant \beta_{33} \int_{0}^{1}\left(|v|^{2}|w|+|w|^{2}|v|\right) d x .
$$

Then, collecting inequalities (4.59), (4.62), and (4.63) together, we conclude that if $|u|_{0}+|v|_{0} \leqslant \delta_{3}:=\min \left\{\delta_{31}, \delta_{32}, \delta_{33}\right\}$ and $\beta_{3}:=\max \left\{\beta_{32}, \beta_{33}\right\}$, then

$$
\dot{V}_{3}=\mathcal{T}_{31}+\mathcal{T}_{32}+\mathcal{T}_{33} \leqslant-\alpha_{3} V_{3}+\beta_{3}\left|v_{1}\right|^{4}+\beta_{3} \int_{0}^{1}\left(|v|^{2}|w|+|w|^{2}|v|\right) d x .
$$

This completes the proof of Lemma 4.5.

Finally, we deal with $V$ (see (4.5)) and $\dot{V}$, which are now considered as functionals on the set $\mathcal{V}$ of $u \in C^{3}\left([0,1], \mathbb{R}^{n}\right)$ satisfying (4.49), (4.51), (4.52), and (4.53) with $v:=$ $u_{x}$ and $w:=u_{x x}, u_{0}:=u(0), u_{1}:=u(1), v_{0}:=u_{x}(0), v_{1}:=u_{x}(1), w_{0}:=u_{x x}(0)$, and $w_{1}:=u_{x x}(1)$. Of course, we "define" $\dot{V}$ by $\dot{V}(u):=\dot{V}_{1}(u)+\dot{V}_{2}\left(u, u_{x}\right)+\dot{V}_{3}\left(u, u_{x}, u_{x x}\right)$. The following lemma holds.

Lemma 4.6. Let $\mu \in\left(0, \min \left\{\mu_{1}, \mu_{2}, \mu_{3}\right\}\right)$. There exist positive real constants $\alpha$, $\beta$, and $\delta$ such that, for every $u \in \mathcal{V}$ such that $|u|_{0}+\left|u_{x}\right|_{0} \leqslant \delta$, we have

$$
\begin{aligned}
\frac{1}{\beta} \int_{0}^{1}\left(|u|^{2}+\left|u_{x}\right|^{2}+\left|u_{x x}\right|^{2}\right) d x & \leqslant V(u) \leqslant \beta \int_{0}^{1}\left(|u|^{2}+\left|u_{x}\right|^{2}+\left|u_{x x}\right|^{2}\right) d x, \\
\dot{V} & \leqslant-\alpha V .
\end{aligned}
$$

Proof of Lemma 4.6. Throughout this proof, $u$ is assumed to be in $\mathcal{V}$. Let $\bar{\delta}:=\min \left\{\delta_{1}, \delta_{2}, \delta_{3}\right\}, \bar{\alpha}:=\min \left\{\alpha_{1}, \alpha_{2}, \alpha_{3}\right\}$, and $\bar{\beta}:=\max \left\{\beta_{1}, \beta_{2}, \beta_{3}\right\}$. It readily follows from (4.11), (4.32), and (4.54) that if $|u|_{0}+\left|u_{x}\right|_{0} \leqslant \bar{\delta}$, then

$$
\frac{1}{\bar{\beta}} \int_{0}^{1}\left(|u|^{2}+\left|u_{x}\right|^{2}+\left|u_{x x}\right|^{2}\right) d x \leqslant V(u) \leqslant \bar{\beta} \int_{0}^{1}\left(|u|^{2}+\left|u_{x}\right|^{2}+\left|u_{x x}\right|^{2}\right) d x .
$$

Copyright (? by SIAM. Unauthorized reproduction of this article is prohibited. 
In order to check (4.66) (for $\delta>0$ small enough and $\beta>0$ large enough), let us first point out that, for every $\eta>0$,

$$
\begin{aligned}
\int_{0}^{1}\left|u_{x}\right|^{2}\left|u_{x x}\right| d x & \leqslant \int_{0}^{1}\left(\frac{1}{4 \eta}\left|u_{x}\right|^{4}+\eta\left|u_{x x}\right|^{2}\right) d x \\
& \leqslant \frac{1}{4 \eta}\left|u_{x}\right|_{0}^{2} \int_{0}^{1}\left|u_{x}\right|^{2} d x+\eta \int_{0}^{1}\left|u_{x x}\right|^{2} d x .
\end{aligned}
$$

In order to get (4.66), it suffices to use (4.12), (4.33), (4.54), (4.55), (4.67), (4.68) with $\eta:=\alpha_{3} /\left(2 \beta_{3}\right)^{2}$ and to point out that

$$
\begin{gathered}
\int_{0}^{1}|u|^{2}\left|u_{x}\right| d x \leqslant\left|u_{x}\right|_{0} \int_{0}^{1}|u|^{2} d x, \\
\int_{0}^{1}\left|u_{x}\right|^{3} d x \leqslant\left|u_{x}\right|_{0} \int_{0}^{1}\left|u_{x}\right|^{2} d x, \\
\int_{0}^{1}\left|u_{x x}\right|^{2}\left|u_{x}\right| d x \leqslant\left|u_{x}\right|_{0} \int_{0}^{1}\left|u_{x x}\right|^{2} d x .
\end{gathered}
$$

This concludes the proof of Lemma 4.6.

Finally, let us explain how to deduce Theorem 2.3 from Proposition 2.1 and Lemma 4.6. By the Sobolev inequality (see, for instance, [3, Théorème VII, page $129])$, there exists $C>0$ such that, for every $u$ in the Sobolev space $H^{2}\left((0,1), \mathbb{R}^{n}\right)$,

$$
|u|_{0}+\left|u_{x}\right|_{0} \leqslant C_{0}|u|_{H^{2}\left((0,1), \mathbb{R}^{n}\right)},
$$

with

$$
|u|_{H^{2}\left((0,1), \mathbb{R}^{n}\right)}:=\left(\int_{0}^{1}\left(|u|^{2}+\left|u_{x}\right|^{2}+\left|u_{x x}\right|^{2}\right) d x\right)^{1 / 2} .
$$

We choose $\mu \in\left(0, \min \left\{\mu_{1}, \mu_{2}, \mu_{3}\right\}\right]$. Let us point out that a simple density argument shows that (4.65) and (4.66) hold for every $u \in H^{2}\left((0,1), \mathbb{R}^{n}\right)$ satisfying (4.51), (4.52), and $|u|_{0}+\left|u_{x}\right|_{0} \leqslant \delta$. Let

$$
\varepsilon:=\min \left\{\frac{\delta}{2 C_{0} \beta}, \frac{\delta_{0}}{\beta}\right\}
$$

Note that $\beta \geqslant 1$. Using Lemma 4.6, (4.69), and (4.70), the following implications hold for every $u \in H^{2}\left((0,1), \mathbb{R}^{n}\right)$ satisfying (4.51) and (4.52):

$$
\left(|u|_{H^{2}\left((0,1), \mathbb{R}^{n}\right)} \leqslant \varepsilon\right) \Rightarrow\left(|u|_{0}+\left|u_{x}\right|_{0} \leqslant \frac{\delta}{2} \text { and } V(u) \leqslant \beta \varepsilon^{2}\right),
$$

$$
\begin{gathered}
\left(|u|_{0}+\left|u_{x}\right|_{0} \leqslant \delta \text { and } V(u) \leqslant \beta \varepsilon^{2}\right) \Rightarrow\left(|u|_{0}+\left|u_{x}\right|_{0} \leqslant \frac{\delta}{2} \text { and }|u|_{H^{2}\left((0,1), \mathbb{R}^{n}\right)} \leqslant \delta_{0}\right), \\
(4.73) \quad\left(|u|_{0}+\left|u_{x}\right|_{0} \leqslant \delta\right) \Rightarrow(\dot{V}(u) \leqslant 0) .
\end{gathered}
$$

Copyright $@$ by SIAM. Unauthorized reproduction of this article is prohibited. 
Now let $u^{0} \in H^{2}\left((0,1), \mathbb{R}^{n}\right)$ satisfying (4.2), (4.3), and

$$
\left|u^{0}\right|_{H^{2}\left((0,1), \mathbb{R}^{n}\right)} \leqslant \varepsilon .
$$

Let $u \in C^{0}\left([0, T), H^{2}\left((0,1), \mathbb{R}^{n}\right)\right)$ be the maximal classical solution the Cauchy problem (2.5)-(2.7). Using implications (4.71) to (4.73), one gets that

$$
\begin{array}{cc}
|u(t, \cdot)|_{H^{2}\left((0,1), \mathbb{R}^{n}\right)} \leqslant \delta_{0} & \forall t \in[0, T), \\
|u(t, \cdot)|_{0}+\left|u_{x}(t, \cdot)\right|_{0} \leqslant \delta & \forall t \in[0, T) .
\end{array}
$$

Using Proposition 2.1 and (4.74), one gets that $T=+\infty$. Using Lemma 4.6 and (4.75), one gets that

$$
|u(t, \cdot)|_{H^{2}\left((0,1), \mathbb{R}^{n}\right)}^{2} \leqslant \beta V(u(t, \cdot)) \leqslant \beta V\left(u^{0}\right) e^{-\alpha t} \leqslant \beta^{2}\left|u^{0}\right|_{H^{2}\left((0,1), \mathbb{R}^{n}\right)}^{2} e^{-\alpha t} .
$$

This concludes the proof of Theorem 2.3 when $m=n$.

Let us now explain the modifications we use in order to deal with the case $0<$ $m<n$ (of course, the case $m=0$ can be reduced to the case $m=n$ by considering $\tilde{u}(t, x):=u(t, 1-x))$.

One first needs the following parametric version of Lemma 4.1.

Lemma 4.7. Let $\Lambda:=\operatorname{diag}\left(\Lambda_{1}, \ldots, \Lambda_{n}\right) \in \mathcal{D}_{n}$ be such that (2.4) holds. There exist a positive real number $\eta$ and a map $\mathcal{N}:\left\{M \times \Delta \in \mathcal{M}_{n, n}(\mathbb{R}) \times \mathcal{D}_{n} ;\|M-\Lambda\|<\right.$ $\eta\} \rightarrow \mathcal{S}_{n}$ of class $C^{\infty}$ such that

$$
\mathcal{N}(\Lambda, \Delta)=\Delta \quad \forall \Delta \in \mathcal{D}_{n}^{\rho},
$$

$\mathcal{N}(M, \Delta) M-M^{\operatorname{tr}} \mathcal{N}(M, \Delta)=0 \quad \forall(M, \Delta) \in \mathcal{M}_{n, n}(\mathbb{R}) \times \mathcal{D}_{n}$ such that $\|M-\Lambda\|<\eta$.

Proof of Lemma 4.7. With the notation of the proof of Lemma 4.1, it suffices to define $\mathcal{N}(M, D)$ by $\mathcal{N}(M, D):=\mathcal{L}_{M}^{-1}(0, \Delta)$.

The Lyapunov function $V$ now has the following structure:

$$
V(u, v, w)=V_{1}(u)+V_{2}(u, v)+V_{3}(u, v, w),
$$

with

$$
\begin{aligned}
& V_{1}(u)=\int_{0}^{1} u^{\operatorname{tr}} Q(x, u) u d x \\
& V_{2}(u, v)=\int_{0}^{1} v^{\operatorname{tr}} R(x, u) v d x \\
& V_{3}(u, v, w)=\int_{0}^{1} w^{\operatorname{tr}} S(x, u) w d x,
\end{aligned}
$$

where $Q(x, u), R(x, u)$, and $S(x, u)$ are symmetric positive definite matrices depending on $x \in[0,1]$ defined in the following way. We fix $D \in \mathcal{D}_{n,+}$ such that $\left\|D K D^{-1}\right\|<1$. Let $\mu \in(0,+\infty)$, which will be chosen small enough later. Let us recall that $|\Lambda|=$ $\operatorname{diag}\left(\left|\Lambda_{1}\right|, \ldots,\left|\Lambda_{n}\right|\right)=\operatorname{diag}\left(\Lambda_{1}, \ldots, \Lambda_{m},\left|\Lambda_{m+1}\right|, \ldots,\left|\Lambda_{n}\right|\right)$.

(i) We define $Q(x, u)$ by

$$
Q(x, u):=\mathcal{N}\left(F(u), D^{2}|\Lambda|^{-1} \operatorname{diag}\left(e^{-\mu x}, \ldots, e^{-\mu x}, e^{\mu x}, \ldots, e^{\mu x}\right)\right) .
$$

Copyright $($ ) by SIAM. Unauthorized reproduction of this article is prohibited. 
(ii) We define $R(x, u)$ by

$$
R(x, u):=\mathcal{N}\left(F(u), D^{2}|\Lambda| \operatorname{diag}\left(e^{-\mu x}, \ldots, e^{-\mu x}, e^{\mu x}, \ldots, e^{\mu x}\right)\right) .
$$

(iii) Finally, we define $R(x, u)$ by

$$
S(x, u):=\mathcal{N}\left(F(u), D^{2}|\Lambda|^{3} \operatorname{diag}\left(e^{-\mu x}, \ldots, e^{-\mu x}, e^{\mu x}, \ldots, e^{\mu x}\right)\right) .
$$

(In the above equalities and in the following, in $\operatorname{diag}\left(e^{-\mu x}, \ldots, e^{-\mu x}, e^{\mu x}, \ldots, e^{\mu x}\right)$, $e^{-\mu x}$ is repeated $m$ times and $e^{\mu x}$ is repeated $(n-m)$ times.) In order to deal with the boundary conditions on $u$ and $v$, let us define

$$
a_{0}:=\left(\begin{array}{l}
u_{+}(0) \\
u_{-}(1)
\end{array}\right), \quad a_{1}:=\left(\begin{array}{l}
u_{+}(1) \\
u_{-}(0)
\end{array}\right), \quad b_{0}:=\left(\begin{array}{l}
v_{+}(0) \\
v_{-}(1)
\end{array}\right), \quad b_{1}:=\left(\begin{array}{l}
v_{+}(1) \\
v_{-}(0)
\end{array}\right) .
$$

The boundary condition (4.10) is now (see (2.6))

$$
a_{0}=G\left(a_{1}\right) .
$$

Now $\mathcal{V}_{1}$ is defined as the set of $u \in C^{1}\left([0,1], \mathbb{R}^{n}\right)$ such that (4.81) holds and $|u|_{0}<\varepsilon_{0}$.

Clearly, the estimate on $V_{1}$ given in Lemma 4.3 still holds. Let us check that the estimate of this lemma on $\dot{V}_{1}$ also holds.

The decomposition (4.15)-(4.18) becomes

$$
\dot{V}_{1}=\mathcal{T}_{11}+\mathcal{T}_{12}+\mathcal{T}_{13}
$$

with

$$
\begin{aligned}
& \mathcal{T}_{11}:=\int_{0}^{1} u^{\operatorname{tr}} Q_{x}(x, u) F(u) u d x \\
& \mathcal{T}_{12}:=-\int_{0}^{1}\left(u^{\operatorname{tr}} Q(x, u) F(u) u\right)_{x} d x \\
& \mathcal{T}_{13}:=\int_{0}^{1} u^{\operatorname{tr}}\left(\left[Q_{u}^{\prime}(x, u) v\right] F(u)+Q(x, u)\left[F^{\prime}(u) v\right]-\left[Q_{u}^{\prime}(x, u) F(u) v\right]\right) u d x .
\end{aligned}
$$

Noticing that

$$
\left.Q_{x}(x, 0)=-\mu D^{2} \Lambda^{-1} \operatorname{diag}\left(e^{-\mu x}, \ldots, e^{-\mu x}, e^{\mu x}, \ldots, e^{\mu x}\right)\right),
$$

the term $\mathcal{T}_{11}$ can be treated as above. Similarly the term $\mathcal{T}_{13}$ can also be treated as above. Concerning $\mathcal{T}_{12}$, one has

$$
\begin{aligned}
\mathcal{T}_{12} & =-u_{1} Q\left(1, u_{1}\right) F\left(u_{1}\right) u_{1}+u_{0} Q\left(0, u_{0}\right) F\left(u_{0}\right) u_{0} \\
& =-u_{1}^{\operatorname{tr}} D^{2}|\Lambda|^{-1} \Lambda u_{1}+u_{0}^{\operatorname{tr}} D^{2}|\Lambda|^{-1} \Lambda u_{0}+\mathcal{O}\left(\left|u_{1}\right|^{3} ;\left|u_{1}\right|\right)+\mathcal{O}\left(\mu\left|u_{1}\right|^{2} ; \mu\right) .
\end{aligned}
$$

Let

$$
\begin{aligned}
K_{++} \in \mathcal{M}_{m, m}(\mathbb{R}), & K_{+-} \in \mathcal{M}_{m,(n-m)}(\mathbb{R}), \\
K_{-+} \in \mathcal{M}_{(n-m), n}(\mathbb{R}), & K_{--} \in \mathcal{M}_{(n-m),(n-m)}(\mathbb{R})
\end{aligned}
$$

Copyright $@$ by SIAM. Unauthorized reproduction of this article is prohibited. 
be such that

$$
K=\left(\begin{array}{ll}
K_{++} & K_{+-} \\
K_{-+} & K_{--}
\end{array}\right) .
$$

Using (4.80) and (4.81), one has

$u_{0}=\left(\begin{array}{cc}K_{++} & K_{+-} \\ 0 & \operatorname{Id}_{n-m}\end{array}\right) a_{1}+\mathcal{O}\left(\left|a_{1}\right|^{2} ;\left|a_{1}\right|\right), \quad u_{1}=\left(\begin{array}{cc}\operatorname{Id}_{m} & 0 \\ K_{-+} & K_{--}\end{array}\right) a_{1}+\mathcal{O}\left(\left|a_{1}\right|^{2} ;\left|a_{1}\right|\right)$.

Using (4.82) and (4.83), straightforward computations lead to

$$
\mathcal{T}_{12}=-a_{1}^{\operatorname{tr}}\left(D^{2}-K^{\operatorname{tr}} D^{2} K\right) a_{1}+\mathcal{O}\left(\left|a_{1}\right|^{3} ;\left|a_{1}\right|\right)+\mathcal{O}\left(\mu\left|a_{1}\right|^{2} ; \mu\right) .
$$

However, $\left\|D K D^{-1}\right\|<1$ implies (and is in fact equivalent to) the property "the symmetric matrix ${ }^{\operatorname{tr}}\left(D^{2}-K^{\operatorname{tr}} D^{2} K\right)$ is positive definite," which, together with (4.84), implies again the existence of $\delta_{12}>0$ such that (4.22) holds if $\left|a_{1}\right| \leqslant \delta_{12}$. Hence Lemma 4.3 still holds.

Similarly it can be checked that Lemmas 4.4 and 4.5 also hold, except that in (4.33) and (4.55), $\left|v_{1}\right|$ has to be replaced by $\left|b_{1}\right|$ (and the definitions of $\mathcal{V}_{2}$ and $\mathcal{V}_{3}$ have to be modified in order to deal with the new compatibility conditions). The proof of Theorem 2.3 is then completed as in the case $m=n$.

Remark 4.8. One can give a lower bound on the exponential decay in Theorem 2.3. Indeed, it follows from our proof of this theorem that, if $\rho_{1}\left(G^{\prime}(0)\right)<1$, for every $\nu \in\left(0,-\min \left\{\left|\lambda_{1}\right|, \ldots,\left|\lambda_{n}\right|\right\} \ln \left(\rho_{1}\left(G^{\prime}(0)\right)\right)\right)$, there exist $\varepsilon>0$ and $C>0$ such that, for every $u^{0} \in H^{2}\left((0,1), \mathbb{R}^{n}\right)$ satisfying $\left|u^{0}\right|_{H^{2}\left((0,1), \mathbb{R}^{n}\right)} \leqslant \varepsilon$ and the compatibility conditions (2.8)-(2.10), the classical solution $u$ to the Cauchy problem (2.5) $-(2.7)$ is defined on $[0,+\infty)$ and satisfies (2.13).

5. Conclusion and final remarks. We have presented a new sufficient condition on the boundary conditions for the exponential stability of one-dimensional nonlinear hyperbolic systems on a bounded interval. Our analysis relies on the construction of an explicit strict Lyapunov function. Moreover, we have compared our sufficient condition with other known sufficient conditions for nonlinear and linear systems. We conclude the paper with two additional comments.

1. The Lyapunov stability analysis presented in this paper can be extended to nonlinear hyperbolic systems of the form

$$
u_{t}+F(u) u_{x}=h(u),
$$

i.e., systems having a nonzero right-hand side $h(u)$ with the map $h: \mathbb{R}^{n} \rightarrow \mathbb{R}^{n}$ of class $C^{2}$ vanishing at zero $(h(0)=0)$. Our main theorem (Theorem 2.3) can be extended, in a straightforward way, to system (5.1) with boundary conditions (1.4), provided $\left\|h^{\prime}(0)\right\|$ is sufficiently small.

2. For the sake of simplicity, we have assumed throughout the paper that the diagonal matrix $F(0)$ has distinct nonzero diagonal entries. It turns out that this assumption may be slightly relaxed when the matrix $F(u)$ is blockdiagonal. Indeed, in such a case, it is sufficient to assume that the $\Lambda_{i}$ values are different in each block, but different blocks may share identical $\Lambda_{i}$ values. This situation typically occurs when the system $u_{t}+F(u) u_{x}=0$ is a model 
for a network of interconnected $2 \times 2$ hyperbolic systems. Typical examples are hydraulic networks modeled by Saint Venant equations [7], road networks modeled by Aw-Rascle equations [1,8], or pipeline networks modeled by isentropic Euler equations [2].

Appendix A. Some properties of the function $\rho_{\mathbf{1}}$. In this appendix we give some properties which are useful for estimating and computing $\rho_{1}$. Some of these properties are used to prove Proposition 3.7.

Proposition A.1. Let $l \in\{1, \ldots, n-1\}$. Let $K_{1} \in \mathcal{M}_{l, l}(\mathbb{R}), K_{2} \in \mathcal{M}_{l, n-l}(\mathbb{R})$, $K_{3} \in \mathcal{M}_{n-l, l}(\mathbb{R}), K_{4} \in \mathcal{M}_{n-l, n-l}(\mathbb{R})$ and let $K \in \mathcal{M}_{n, n}(\mathbb{R})$ be defined by

$$
K:=\left(\begin{array}{ll}
K_{1} & K_{2} \\
K_{3} & K_{4}
\end{array}\right) .
$$

Then

$$
\rho_{1}(K) \geqslant \max \left\{\rho_{1}\left(K_{1}\right), \rho_{1}\left(K_{4}\right)\right\} .
$$

Moreover, if $K_{2}=0$ or $K_{3}=0$, then

$$
\rho_{1}(K)=\max \left\{\rho_{1}\left(K_{1}\right), \rho_{1}\left(K_{4}\right)\right\} .
$$

Proof of Proposition A.1. Let $D \in \mathcal{D}_{n,+}$. Let $D_{1} \in \mathcal{D}_{l,+}$ and $D_{2} \in \mathcal{D}_{n-l,+}$ be such that

$$
D=\left(\begin{array}{cc}
D_{1} & 0 \\
0 & D_{2}
\end{array}\right) \text {. }
$$

Let

$$
M:=D K D^{-1}
$$

We have

$$
M^{\operatorname{tr}} M=\left(\begin{array}{ll}
M_{11} & M_{12} \\
M_{21} & M_{22}
\end{array}\right)
$$

with

$$
\begin{aligned}
& M_{11}:=D_{1}^{-1} K_{1}^{\mathrm{tr}} D_{1}^{2} K_{1} D_{1}^{-1}+D_{1}^{-1} K_{3}^{\mathrm{tr}} D_{2}^{2} K_{3} D_{1}^{-1}, \\
& M_{12}:=D_{1}^{-1} K_{1}^{\mathrm{tr}} D_{1}^{2} K_{2} D_{2}^{-1}+D_{1}^{-1} K_{3}^{\mathrm{tr}} D_{2}^{2} K_{4} D_{2}^{-1}, \\
& M_{21}:=D_{2}^{-1} K_{2}^{\mathrm{tr}} D_{1}^{2} K_{1} D_{1}^{-1}+D_{2}^{-1} K_{4}^{\mathrm{tr}} D_{2}^{2} K_{3} D_{1}^{-1}, \\
& M_{22}:=D_{2}^{-1} K_{2}^{\mathrm{tr}} D_{1}^{2} K_{2} D_{2}^{-1}+D_{2}^{-1} K_{4}^{\mathrm{tr}} D_{2}^{2} K_{4} D_{2}^{-1} .
\end{aligned}
$$

For $X \in \mathbb{R}^{l}$, let $\tilde{X} \in \mathbb{R}^{n}$ be defined by

$$
\tilde{X}:=\left(\begin{array}{c}
X \\
0
\end{array}\right) .
$$

Copyright $\odot$ by SIAM. Unauthorized reproduction of this article is prohibited. 
Note that $|\tilde{X}|=|X|$ and that

$$
\begin{aligned}
\tilde{X}^{\operatorname{tr}} M^{\operatorname{tr}} M \tilde{X} & =X^{\operatorname{tr}} D_{1}^{-1} K_{1}^{\operatorname{tr}} D_{1}^{2} K_{1} D_{1}^{-1} X+X^{\operatorname{tr}} D_{1}^{-1} K_{3}^{\operatorname{tr}} D_{2}^{2} K_{3} D_{1}^{-1} X \\
& \geqslant X^{\operatorname{tr}} D_{1}^{-1} K_{1}^{\operatorname{tr}} D_{1}^{2} K_{1} D_{1}^{-1} X .
\end{aligned}
$$

Hence

$$
\begin{aligned}
\max \left\{Z^{\operatorname{tr}} M^{\operatorname{tr}} Z ; Z \in \mathbb{R}^{n},|Z|=1\right\} & \geqslant \max \left\{X^{\operatorname{tr}} D_{1}^{-1} K_{1}^{\operatorname{tr}} D_{1}^{2} K_{1} D_{1}^{-1} X ; X \in \mathbb{R}^{l},|X|=1\right\} \\
& \geqslant \rho_{1}\left(K_{1}\right)^{2},
\end{aligned}
$$

which implies that $\rho_{1}\left(K_{1}\right) \leqslant \rho_{1}(K)$. Similarly $\rho_{1}\left(K_{4}\right) \leqslant \rho_{1}(K)$. This proves (A.1).

Let us now prove (A.2). We deal only with the case $K_{3}=0$ (the case $K_{2}=0$ being similar). Let $\eta>0$. Let $D_{1} \in \mathcal{D}_{l,+}$ and $D_{2} \in \mathcal{D}_{n-l,+}$ be such that

$$
\left\|D_{1} K_{1} D_{1}^{-1}\right\| \leqslant \rho_{1}\left(K_{1}\right)+\eta, \quad\left\|D_{2} K_{4} D_{2}^{-1}\right\| \leqslant \rho_{1}\left(K_{4}\right)+\eta .
$$

Let $\varepsilon>0$ and

$$
D:=\left(\begin{array}{cc}
\varepsilon D_{1} & 0 \\
0 & D_{2}
\end{array}\right) \in \mathcal{D}_{n,+}, \quad M:=D K D^{-1} \in \mathcal{M}_{n, n}(\mathbb{R}) .
$$

Let $Z \in \mathbb{R}^{n}$ and let $X \in \mathbb{R}^{l}$ and $Y \in \mathbb{R}^{n-l}$ be such that

$$
Z=\left(\begin{array}{l}
X \\
Y
\end{array}\right)
$$

We have

$$
\begin{aligned}
Z^{\operatorname{tr}} M^{\operatorname{tr}} M Z= & X^{\operatorname{tr}} D_{1}^{-1} K_{1}^{\operatorname{tr}} D_{1}^{2} K_{1} D_{1}^{-1} X+2 \varepsilon X^{\operatorname{tr}} D_{1}^{-1} K_{1}^{\operatorname{tr}} D_{1}^{2} K_{2} D_{2}^{-1} Y \\
& +\varepsilon^{2} Y^{\operatorname{tr}} D_{2}^{-1} K_{2}^{\operatorname{tr}} D_{1}^{2} K_{2} D_{2}^{-1} Y+Y^{\operatorname{tr}} D_{2}^{-1} K_{4}^{\operatorname{tr}} D_{2}^{2} K_{4} D_{2}^{-1} Y .
\end{aligned}
$$

Hence there exists a constant $C>0$ independent of $Z$ and $\varepsilon>0$ such that

$$
Z^{\operatorname{tr}} M^{\operatorname{tr}} M Z \leqslant\left(\left\|D_{1} K_{1} D_{1}^{-1}\right\||X|\right)^{2}+\left(\left\|D_{2} K_{4} D_{2}^{-1}\right\||Y|\right)^{2}+C \varepsilon|Z|^{2} .
$$

From (2.1), (A.3), and (A.4), we obtain that

$$
\rho_{1}(K)^{2} \leqslant \max \left\{\left(\rho_{1}\left(K_{1}\right)+\eta\right)^{2},\left(\rho_{1}\left(K_{4}\right)+\eta\right)^{2}\right\}+C \varepsilon .
$$

Letting $\varepsilon \rightarrow 0$ and $\eta \rightarrow 0$ in (A.5), one gets that $\rho_{1}(K)^{2} \leqslant \max \left\{\rho_{1}\left(K_{1}\right)^{2}, \rho_{1}\left(K_{4}\right)^{2}\right\}$. This concludes the proof of Proposition A.1.

Proposition A.2. The map $\rho_{1}: \mathcal{M}_{n, n}(\mathbb{R}) \rightarrow[0,+\infty)$ is continuous.

Proof of Proposition A.2. We proceed by induction on $n$. For $n=1$ the function $\rho_{1}$ satisfies $\rho(k)=|k|$ for every $k \in \mathbb{R}=\mathcal{M}_{1,1}(\mathbb{R})$ and is therefore continuous. We now assume that $\rho_{1}$ is continuous on $\mathcal{M}_{p, p}(\mathbb{R})$ for every $p \in\{1, \ldots, n-1\}$ and prove that $\rho_{1}$ is continuous on $\mathcal{M}_{n, n}(\mathbb{R})$. Since, for every $D \in \mathcal{D}_{n,+}$, the function $K \in \mathcal{M}_{n, n}(\mathbb{R}) \mapsto\|K\| \in \mathbb{R}$ is continuous, it readily follows from (2.1) that $\rho_{1}$ is upper semicontinuous on $\mathcal{M}_{n, n}(\mathbb{R})$. It remains only to check that $\rho_{1}$ is lower semicontinuous.

Copyright (C) by SIAM. Unauthorized reproduction of this article is prohibited. 
We argue by contradiction: let $K \in \mathcal{D}_{n, n}(\mathbb{R})$ and let $\left(K_{k}\right)_{k \in \mathbb{N}}$ be a sequence of elements of $\mathcal{M}_{n, n}(\mathbb{R})$ such that

$$
\begin{aligned}
& K_{k} \rightarrow K \text { as } k \rightarrow+\infty, \\
& \lim _{k \rightarrow+\infty} \rho_{1}\left(K_{k}\right)<\rho_{1}(K) .
\end{aligned}
$$

Let $\left(D_{k}\right)_{k \in \mathbb{N}}$ be a sequence of elements of $\mathcal{D}_{n,+}$ such that

$$
\left\|D_{k} K_{k} D_{k}^{-1}\right\| \leqslant \rho_{1}\left(K_{k}\right)+k^{-1} \quad \forall k \in \mathbb{N} \backslash\{0\} .
$$

Note that, denoting by $\left(e_{1}, \ldots, e_{n}\right)$ the canonical basis of $\mathbb{R}^{n}$,

$$
\left|A_{i j}\right|=\left|e_{i}^{\mathrm{tr}} A e_{j}\right| \leqslant\|A\| \quad \forall A \in \mathcal{M}_{n, n}(\mathbb{R}), \forall i \in\{1, \ldots, n\}, \forall j \in\{1, \ldots, n\} .
$$

Hence, if we denote by $K_{i j k}$ the term on the $i$ th line and $j$ th column of the matrix $K_{k}$,

$$
\left|K_{i j k}\right| \frac{d_{i k}}{d_{j k}} \leqslant\left\|D_{k} K_{k} D_{k}^{-1}\right\| \quad \forall(i, j) \in\{1, \ldots, n\}^{2}, \forall k \in \mathbb{N},
$$

where $\left(d_{i k}\right)_{i \in\{1, \ldots, n\}}$ is defined by $D_{k}=\operatorname{diag}\left(d_{1 k}, \ldots, d_{n k}\right)$. After suitable reorderings (note that $\rho_{1}\left(\Sigma A \Sigma^{-1}\right)=\rho_{1}(A)$ for every $A \in \mathcal{M}_{n, n}(\mathbb{R})$ and for every permutation matrix $\Sigma$ ) and extracting subsequences if necessary, we may assume without loss of generality that

$$
d_{1 k} \leqslant d_{2 k} \leqslant \cdots \leqslant d_{(n-1) k} \leqslant d_{n k} \quad \forall k \in \mathbb{N} .
$$

A simple scaling argument also shows that we may assume without loss of generality that

$$
d_{1 k}=1 \quad \forall k \in \mathbb{N} .
$$

Extracting subsequences if necessary, there exist $l \in\{1, \ldots, n\},\left(d_{1}, \ldots, d_{l}\right) \in[1,+\infty)^{l}$ such that

$$
\begin{gathered}
d_{i k} \rightarrow d_{i} \text { as } k \rightarrow+\infty \quad \forall i \in\{1, \ldots, l\}, \\
d_{i k} \rightarrow+\infty \text { as } k \rightarrow+\infty \quad \forall i \in\{l+1, \ldots, n\} .
\end{gathered}
$$

We first treat the case where $l=n$. Let $D:=\operatorname{diag}\left(d_{1}, \ldots, d_{n}\right) \in \mathcal{D}_{n,+}$. From (A.12), we have

$$
D_{k} \rightarrow D \text { as } k \rightarrow+\infty \text {. }
$$

From (2.1), we have

$$
\rho_{1}(K) \leqslant\left\|D K D^{-1}\right\|,
$$

which, together with (A.8) and (A.14), implies that

$$
\liminf _{k \rightarrow+\infty} \rho_{1}\left(K_{k}\right) \geqslant \rho_{1}(K),
$$

in contradiction with (A.7). 
It remains to deal with the case where $l<n$. Let us denote $K_{i j}$ the term on the $i$ th line and $j$ th column of the matrix $K$. From (A.6), (A.7), (A.8), (A.9), (A.12), and (A.13), one gets that

$$
K_{i j}=0 \quad \forall(i, j) \in\{l+1, \ldots, n\} \times\{1, \ldots, l\} .
$$

Let $K^{1} \in \mathcal{M}_{l, l}(\mathbb{R}), K^{2} \in \mathcal{M}_{l, n-l}(\mathbb{R}), K^{4} \in \mathcal{M}_{n-l, n-l}(\mathbb{R})$ be such that

$$
K=\left(\begin{array}{cc}
K^{1} & K^{2} \\
0 & K^{4}
\end{array}\right)
$$

Similarly, for $k \in \mathbb{N}$, let $K_{k}^{1} \in \mathcal{M}_{l, l}(\mathbb{R}), K_{k}^{2} \in \mathcal{M}_{l, n-l}(\mathbb{R}), K_{k}^{3} \in \mathcal{M}_{n-l, l}(\mathbb{R}), K_{k}^{4} \in$ $\mathcal{M}_{n-l, n-l}(\mathbb{R})$ be defined by

$$
K:=\left(\begin{array}{cc}
K_{k}^{1} & K_{k}^{2} \\
K_{k}^{3} & K_{k}^{4}
\end{array}\right)
$$

From (A.2), we have

$$
\rho_{1}(K)=\max \left\{\rho_{1}\left(K^{1}\right), \rho_{1}\left(K^{4}\right)\right\}
$$

From (A.1), we have

$$
\rho_{1}\left(K_{k}\right) \geqslant \max \left\{\rho_{1}\left(K_{k}^{1}\right), \rho_{1}\left(K_{k}^{4}\right)\right\} \quad \forall k \in \mathbb{N} .
$$

From our induction hypothesis (the continuity of $\rho_{1}$ on $\mathcal{M}_{p, p}(\mathbb{R})$ for every $p \in$ $\{1, \ldots, n-1\})$ and (A.6), we get that

$$
\lim _{k \rightarrow+\infty} \rho_{1}\left(K_{k}^{1}\right)=\rho_{1}\left(K^{1}\right), \quad \lim _{k \rightarrow+\infty} \rho_{1}\left(K_{k}^{4}\right)=\rho_{1}\left(K^{4}\right),
$$

which, together with (A.17) and (A.18), again leads to a contradiction with (A.7). This concludes the proof of Proposition A.2.

Our next proposition shows a case where the value of $\rho_{1}(K)$ may be given directly. (For a converse of this proposition, see Proposition B.1.)

Proposition A.3. Let $l \in\{1, \ldots, n\}$. Let $\left(A_{j}\right)_{j \in\{1, \ldots, l\}}$ and $\left(B_{j}\right)_{j \in\{1, \ldots, l\}}$ be two sequences of vectors in $\mathbb{R}^{n}$ such that

$$
\begin{gathered}
A_{j}^{\operatorname{tr}} A_{k}=B_{j}^{\operatorname{tr}} B_{k} \quad \forall(j, k) \in\{1, \ldots, l\}^{2}, \\
\sum_{j=1}^{l} A_{i j}^{2}=\sum_{j=1}^{l} B_{i j}^{2} \quad \forall i \in\{1, \ldots, n\},
\end{gathered}
$$

where $A_{i j}$ (resp., $B_{i j}$ ) is the element on the ith line of the vector $A_{j}$ (resp., $B_{j}$ ). We assume that the $l$ vectors $A_{1}, \ldots, A_{l}$ are linearly independent. Let $R \geqslant 0$ and let $K \in \mathcal{M}_{n, n}(\mathbb{R})$ be such that

$$
\begin{gathered}
K A_{j}=R B_{j} \quad \forall j \in\{1, \ldots, l\}, \\
|K X| \leqslant R|X| \quad \forall X \in \mathbb{R}^{n} \text { such that } X^{\operatorname{tr}} A_{j}=0 \quad \forall j \in\{1, l\} .
\end{gathered}
$$

Then $\rho_{1}(K)=R$. 
Proof of Proposition A.3. It readily follows from the assumptions of this proposition that $\|K\|=R$. Hence it remains only to check that

$$
\left\|D K D^{-1}\right\| \geqslant R \quad \forall D \in \mathcal{D}_{n,+} .
$$

Let $D:=\operatorname{diag}\left(D_{1}, \ldots, D_{n}\right) \in \mathcal{D}_{n,+}$. For $j \in\{1, \ldots, l\}$, let us define

$$
E_{j}:=\left(E_{1 j}, \ldots, E_{n j}\right)^{\operatorname{tr}} \in \mathbb{R}^{n} \backslash\{0\}, \quad F_{j}:=\left(F_{1 j}, \ldots, F_{n j}\right)^{\operatorname{tr}} \in \mathbb{R}^{n} \backslash\{0\}
$$

by

$$
E_{j}:=D A_{j}, \quad F_{j}:=D B_{j} .
$$

We have, for every $j \in\{1, \ldots, l\}$,

$$
\begin{gathered}
D K D^{-1} E_{j}=R F_{j}, \\
E_{i j}=D_{i} A_{i j}, \quad F_{i j}=D_{i} B_{i j} \quad \forall i \in\{1, \ldots, n\} .
\end{gathered}
$$

Using (A.20), (A.24), and (A.25), we get

$$
\begin{aligned}
\sum_{j=1}^{l}\left|D K D^{-1} E_{j}\right|^{2} & =R^{2} \sum_{j=1}^{l}\left(\sum_{i=1}^{n} F_{i j}^{2}\right) \\
& =R^{2} \sum_{i=1}^{n} D_{i}^{2}\left(\sum_{j=1}^{l} B_{i j}^{2}\right) \\
& =R^{2} \sum_{i=1}^{n} D_{i}^{2}\left(\sum_{j=1}^{l} A_{i j}^{2}\right) \\
& =R^{2} \sum_{j=1}^{l}\left|E_{j}\right|^{2} .
\end{aligned}
$$

In particular, there exists $p \in\{1, \ldots l\}$, such that

$$
\left|D K D^{-1} E_{p}\right|^{2} \geqslant R^{2}\left|E_{p}\right|^{2},
$$

which, together with the fact that $E_{p} \neq 0$, implies that $\left\|D K D^{-1}\right\| \geqslant R$. This concludes the proof of Proposition A.3.

Appendix B. Proof of Proposition 3.7. Inequality (3.22) is obvious: indeed, for every $\left(\theta_{1}, \ldots, \theta_{n}\right)^{\operatorname{tr}} \in \mathbb{R}^{n}$ and for every $D \in \mathcal{D}_{n,+}$,

$$
\begin{aligned}
\rho\left(\operatorname{diag}\left(e^{\iota \theta_{1}}, \ldots, e^{\iota \theta_{n}}\right) K\right) & =\rho\left(D \operatorname{diag}\left(e^{\iota \theta_{1}}, \ldots, e^{\iota \theta_{n}}\right) K D^{-1}\right) \\
& =\rho\left(\operatorname{diag}\left(e^{\iota \theta_{1}}, \ldots, e^{\iota \theta_{n}}\right) D K D^{-1}\right) \\
& \leqslant\left\|\operatorname{diag}\left(e^{\iota \theta_{1}}, \ldots, e^{\iota \theta_{n}}\right) D K D^{-1}\right\| \\
& \leqslant\left\|\operatorname{diag}\left(e^{\iota \theta_{1}}, \ldots, e^{\iota \theta_{n}}\right)\right\|\left\|D K D^{-1}\right\|=\left\|D K D^{-1}\right\| .
\end{aligned}
$$

Copyright $(\odot$ by SIAM. Unauthorized reproduction of this article is prohibited. 
The proof of (3.23) for every $n \in\{1,2,3,4,5\}$ is more complicated and relies on various independent propositions. The first proposition provides the converse (up to the $D$ ) to Proposition A.3 for generic $K \in \mathcal{M}_{n, n}(\mathbb{R})$.

Proposition B.1. Let $K \in \mathcal{M}_{n, n}(\mathbb{R})$ be such that, for every $M>0$, there exists $\delta>0$ such that

$$
\begin{aligned}
\left(D:=\left(D_{1}, \ldots, D_{n}\right) \in \mathcal{D}_{n,+}, \sum_{i=1}^{n} D_{i}=1, \min \left\{D_{1}, \ldots, D_{n}\right\}<\delta\right) & \\
& \Rightarrow\left(\left\|D K D^{-1}\right\|>M\right) .
\end{aligned}
$$

(It is easily checked that this property holds, for example, if $K_{i j} \neq 0$, for every $(i, j) \in$ $\{1, \ldots, n\}^{2}$ such that $i \neq j$, which is a generic property.) Then there exist $D \in \mathcal{D}_{n,+}$, an integer $l \in\{1, \ldots, n\}, l$ vectors $A_{j} \in \mathbb{R}^{n}, j \in\{1, \ldots, l\}$, and l vectors $B_{j} \in \mathbb{R}^{n}$, $j \in\{1, \ldots, l\}$, such that (A.19) and (A.20) hold and

$$
\begin{gathered}
\text { the vectors } A_{j} \in \mathbb{R}^{n}, j \in\{1, \ldots, l\}, \text { are linearly independent, } \\
\qquad \begin{array}{c}
D K D^{-1} A_{j}=\rho_{1}(K) B_{j} \quad \forall j \in\{1, \ldots l\}, \\
\left|D K D^{-1} X\right| \leqslant \rho_{1}(K)|X| \quad \forall X \in \mathbb{R}^{n} .
\end{array}
\end{gathered}
$$

Remark B.2. Proposition B.1 is false if assumption (B.1) is removed. Indeed, let us take $n=2$ and

$$
K=\left(\begin{array}{ll}
0 & 1 \\
0 & 0
\end{array}\right) .
$$

Then $\rho_{1}(K)=0$, and it is easily seen that the conclusion of Proposition B.1 does not hold.

Proof of Proposition B.1. From (B.1), one gets the existence of $\tilde{D} \in \mathcal{D}_{n,+}$ such that

$$
\left\|\tilde{D} K \tilde{D}^{-1}\right\|=\rho_{1}(K)
$$

Replacing $K$ by $\tilde{D} K \tilde{D}^{-1}$, we may assume without loss of generality that $\tilde{D}$ is the identity $\operatorname{map} \operatorname{Id}_{n}$ of $\mathbb{R}^{n}$. Then

$$
\|K\|=\rho_{1}(K) .
$$

Clearly, (B.1) implies that $K \neq 0$, and therefore, by (B.6),

$$
\rho_{1}(K) \neq 0 .
$$

(In fact, if $K=0$, the conclusion of Proposition B.1 obviously holds.) Note that (B.6) implies (B.4) with $D:=\operatorname{Id}_{n}$. Let $p \in\{1, \ldots, n\}$ be the dimension of the kernel of $K^{\text {tr }} K-\rho_{1}(K)^{2} \operatorname{Id}_{n}$ and let $\left(X_{1}, \ldots, X_{p}\right)$ be an orthonormal basis of this kernel. For $j \in\{1, \ldots, p\}$, let $Y_{j}:=K X_{j}$. One has

$$
\left|Y_{j}\right|^{2}=X_{j}^{\operatorname{tr}} K^{\operatorname{tr}} K X_{j}=\rho_{1}(K)^{2}\left|X_{j}\right|^{2} \quad \forall j \in\{1, \ldots, p\},
$$

$$
Y_{k}^{\mathrm{tr}} Y_{j}=X_{k}^{\mathrm{tr}} K^{\mathrm{tr}} K X_{j}=\rho_{1}(K)^{2} X_{k}^{\mathrm{tr}} X_{j}=0 \quad \forall(k, j) \in\{1, \ldots, p\}^{2} \text { such that } k \neq j .
$$


For $i \in\{1, \ldots, n\}$ and $j \in\{1, \ldots, p\}$, let us denote by $X_{i j}$ (resp., $Y_{i j}$ ) the $i$ th component of $X_{j}$ (resp., $Y_{j}$ ). For $j \in\{1, \ldots, p\}$, let us denote by $E_{j}$ the element of $\mathbb{R}^{n}$ whose $i$ th component is

$$
E_{i j}:=Y_{i j}^{2}-X_{i j}^{2}
$$

Let us assume, for the moment, that

$$
\forall \tau \in \mathbb{R}^{n} \text {, there exists } j \in\{1, \ldots, p\} \text { such that } \tau^{\mathrm{tr}} E_{j} \geqslant 0 .
$$

Applying the separation principle for convex sets to $\{0\}$ and the convex hull of the vectors $E_{j}, j \in\{1, \ldots, p\}$ (see, e.g., [19, Theorem 3.4(b), page 58]), it follows from (B.11) that $0 \in \mathbb{R}^{n}$ is in the convex hull of the vectors $E_{1}, \ldots E_{p}$ : there exist $p$ nonnegative real numbers $t_{1}, \ldots, t_{p}$ such that

$$
\sum_{j=1}^{p} t_{i}=1, \quad \sum_{j=1}^{p} t_{i} E_{i}=0 .
$$

Let $l \in\{1, \ldots, p\}$ be the number of the $t_{i}$ 's which are not equal to 0 . Reordering the $X_{i}$ 's if necessary, we may assume that

$$
t_{j}>0 \quad \forall j \in\{1, \ldots, l\}, \quad t_{j}=0 \quad \forall j \in\{l+1, \ldots p\} .
$$

For $j \in\{1, \ldots, l\}$, we define $A_{j} \in \mathbb{R}^{n}$ and $B_{j} \in \mathbb{R}^{n}$ by

$$
A_{j}:=\sqrt{t_{j}} X_{j}, \quad B_{j}:=\sqrt{t_{j}} Y_{j} .
$$

Then it is easily checked that the vectors $A_{1}, \ldots, A_{l}$ are linearly independent, that (A.19) and (A.20) hold (one even has $A_{k}^{\text {tr }} A_{j}=B_{k}^{\text {tr }} B_{j}=0$ for every $(k, j) \in\{1, \ldots, l\}^{2}$ such that $k \neq j$ ), and that (B.3) holds with $D:=\operatorname{Id}_{n}$.

It remains only to prove (B.11). Let $\tau:=\left(\tau_{1}, \ldots, \tau_{n}\right)^{\operatorname{tr}} \in \mathbb{R}^{n}$. For $s \in \mathbb{R}$, let

$$
D(s):=\operatorname{diag}\left(1+s \tau_{1}, \ldots, 1+s \tau_{n}\right) \in \mathcal{D}_{n} .
$$

For $s$ small enough, $D(s) \in \mathcal{D}_{n,+}$, and therefore, by (B.6),

$$
\left\|D(s) K D(s)^{-1}\right\|^{2} \geqslant\|K\|^{2}=\left\|D(0) K D(0)^{-1}\right\|^{2} .
$$

Let us estimate the left-hand side of (B.13). By a classical theorem due to Rellich (see, e.g., [18, Theorem XII.3, page 4]) on perturbations of the spectrum of self-adjoint operators, there exist $\varepsilon>0, p$ real functions $\lambda_{1}, \ldots, \lambda_{p}$ of class $C^{1}$ from $(-\varepsilon, \varepsilon)$ into $\mathbb{R}$, and $p$ maps $x_{1}, \ldots, x_{p}$ of class $C^{1}$ from $(-\varepsilon, \varepsilon)$ into $\mathbb{R}^{n}$ such that

$$
\begin{gathered}
\lambda_{j}(0)=\rho_{1}(K)^{2}, \quad x_{j}(0)=X_{j} \quad \forall j \in\{1, \ldots, p\}, \\
D(s)^{-1} K^{\operatorname{tr}} D(s)^{2} K D(s)^{-1} x_{j}(s)=\lambda_{j}(s) x_{j}(s) \quad \forall s \in(-\varepsilon, \varepsilon), \forall j \in\{1, \ldots, p\}, \\
x_{j}(s)^{\operatorname{tr}} x_{j}(s)=1 \quad \forall s \in(-\varepsilon, \varepsilon), \forall j \in\{1, \ldots, p\}, \\
x_{j}(s)^{\operatorname{tr}} x_{k}(s)=0 \quad \forall s \in(-\varepsilon, \varepsilon), \forall(j, k) \in\{1, \ldots, p\}^{p} \text { such that } k \neq j, \\
\left\|D(s) K D(s)^{-1}\right\|^{2}=\max \left\{\lambda_{1}(s), \ldots, \lambda_{p}(s)\right\} \quad \forall s \in(-\varepsilon, \varepsilon) .
\end{gathered}
$$

Copyright $@$ by SIAM. Unauthorized reproduction of this article is prohibited. 
Differentiating (B.15) with respect to $s$ and using (B.10), (B.14), (B.16), and (B.17), one gets

$$
\lambda_{j}^{\prime}(0)=2 \rho_{1}(K)^{2} \tau^{\mathrm{tr}} E_{j} \quad \forall j \in\{1, \ldots, p\} .
$$

Property (B.11) follows from (B.7), (B.13), (B.18), and (B.19). This concludes the proof of Proposition B.1.

The number $l$ appearing in Proposition B.1 turns out to be important to compare $\rho_{0}$ and $\rho_{1}$ : we have the following proposition.

Proposition B.3. Let $K \in \mathcal{M}_{n, n}(\mathbb{R}), D \in \mathcal{D}_{n,+}, l \in\{1, \ldots, n\}, l$ vectors $A_{j} \in$ $\mathbb{R}^{n}, j \in\{1, \ldots, l\}$, and $l$ vectors $B_{j} \in \mathbb{R}^{n}, j \in\{1, \ldots, l\}$, be such that (A.20), (B.2), (B.3), and (B.4) hold. If $l=1$, there exist $X \in \mathbb{R}^{n}$ and $\Upsilon:=\operatorname{diag}\left(\Upsilon_{1}, \ldots, \Upsilon_{n}\right) \in \mathcal{D}_{n}$ such that

$$
\begin{gathered}
|X| \neq 0, \\
\Upsilon_{i} \in\{1,-1\} \quad \forall i \in\{1, \ldots, n\}, \\
K X=\rho_{1}(K) \Upsilon X .
\end{gathered}
$$

If $l=2$, there exist $X \in \mathbb{C}^{n}$ and $\left(\Upsilon_{1}, \ldots, \Upsilon_{n}\right) \in \mathbb{C}^{n}$ such that

$$
\begin{gathered}
|X| \neq 0, \\
\left|\Upsilon_{i}\right|=1 \quad \forall i \in\{1, \ldots, n\}, \\
K X=\rho_{1}(K) \operatorname{diag}\left(\Upsilon_{1}, \ldots, \Upsilon_{n}\right) X .
\end{gathered}
$$

In both cases $(l=1$ or $l=2)$, one has (3.23).

Proof of Proposition B.3. Let us first consider the case $l=1$. Let $i \in\{1, \ldots, n\}$. From (A.20), one has $\left|A_{i 1}\right|=B_{i 1}$, and therefore there exists $\Upsilon_{i} \in\{-1,1\}$ such that $B_{i 1}=\varepsilon_{i} A_{i 1}$. From (B.3), one gets (B.22) if one defines $X$ by $X:=D^{-1} A_{1}$. Let us check that (3.23) holds. Let $\left(\theta_{1}, \ldots, \theta_{n}\right)^{\operatorname{tr}} \in \mathbb{R}^{n}$ be defined by

$$
\theta_{i}=0 \text { if } \Upsilon_{i}=1, \quad \theta_{i}=-\pi \text { if } \Upsilon_{i}=-1
$$

Then (B.22) implies that

$$
\operatorname{diag}\left(e^{\iota \theta_{1}}, \ldots, e^{\iota \theta_{n}}\right) K X=\rho_{1}(K) X
$$

From (3.21), (B.20), and (B.26), we get that

$$
\rho_{0}(K) \geqslant \rho_{1}(K)
$$

which, together with (3.22), gives (3.23).

Let us now turn to the case $l=2$. Let $i \in\{1, \ldots, n\}$. From (A.20), one has

$$
\left|A_{i 1}+\iota A_{i 2}\right|=\left|B_{i 1}+\iota B_{i 2}\right|
$$

and therefore there exists $\Upsilon_{i} \in \mathbb{C}$ such that $\left|\Upsilon_{i}\right|=1$ and $B_{i 1}+\iota B_{i 2}=\Upsilon_{i}\left(A_{i 1}+\iota A_{i 2}\right)$. From (B.3), one gets (B.22) if one defines $X$ by $X:=D^{-1}\left(A_{1}+\iota A_{2}\right)$. Finally, the proof of (3.23) is the same as in the case $l=1$. This concludes the proof of Proposition B.3.

The next proposition deals with the case $n=l$. 
Proposition B.4. Let $K \in \mathcal{M}_{n, n}(\mathbb{R}), D \in \mathcal{D}_{n,+}, l \in\{1, \ldots, n\}, l$ vectors $A_{j} \in \mathbb{R}^{n}, j \in\{1, \ldots, l\}$, and $l$ vectors $B_{j} \in \mathbb{R}^{n}, j \in\{1, \ldots, l\}$, be such that (A.20), (B.2), (B.3), and (B.4) hold. If $l=n$, there exist $X \in \mathbb{C}^{n}$ satisfying (B.23) and $\theta \in \mathbb{R}$ such that

$$
K X=e^{-\iota \theta} \rho_{1}(K) X
$$

and (3.23) again holds.

Proof of Proposition B.4. If $\rho_{1}(K)=0$, then $K=0$ and the conclusion of Proposition B.4 holds. If $\rho_{1}(K)>0$, it follows from (A.19), (B.2), and (B.3) and the assumption $l=n$ that $\rho_{1}(K)^{-1} K D K D^{-1}$ is an isometry. Hence there exist $Y \in \mathbb{C}^{n} \backslash\{0\}$ and $\theta \in \mathbb{R}$ such that $\rho_{1}(K)^{-1} D K D^{-1} Y=e^{-\iota \theta} Y$, which implies (B.28) if $X:=D^{-1} Y$. Finally, (3.23) again follows from (B.23) and (B.28). This concludes the proof of Proposition B.4.

Note that $\rho_{0}$ is continuous. Hence, from Proposition A.2, Proposition B.1, Proposition B.3, and Proposition B.4, in order to get (3.23) (for every $n \in\{1, \ldots, 5\}$ ) of Proposition 3.7, it remains to address, with the notation of the conclusion of Proposition B.1, the cases $(l, n)=(3,4),(l, n)=(3,5)$, and $(l, n)=(4,5)$. This is done in the following proposition.

Proposition B.5. Let $K \in \mathcal{M}_{n, n}(\mathbb{R}), D \in \mathcal{D}_{n,+}, l \in\{1, \ldots, n\}, l$ vectors $A_{j} \in \mathbb{R}^{n}, j \in\{1, \ldots, l\}$, and l vectors $B_{j} \in \mathbb{R}^{n}, j \in\{1, \ldots, l\}$, be such that (A.20), (B.2), (B.3), and (B.4) hold. If $(l, n) \in\{(3,4),(3,5),(4,5)\}$, there exist $X \in \mathbb{C}^{n}$ and $\left(\Upsilon_{1}, \ldots, \Upsilon_{n}\right) \in \mathbb{C}^{n}$ such that (B.23), (B.24), and (B.25) hold. In particular, one has (3.23).

Proof of Proposition B.5. The fact that (3.23) is implied by the assumptions of Proposition B.5, (B.23), (B.24), and (B.25) has already been pointed out in the proof of Proposition B.3. The case $(l, n)=(3,4)$ follows from the case $(l, n)=(3,5)$ by replacing $K \in \mathcal{M}_{4,4}(\mathbb{R})$ by the matrix

$$
\tilde{K}:=\left(\begin{array}{cc}
K & 0 \\
0 & 0
\end{array}\right)
$$

Hence we may assume that $n=5$. Taking $X:=D^{-1}\left(Y_{1} A_{1}+Y_{2} A_{2}+\cdots+Y_{l} A_{l}\right)$ it suffices to prove the existence of $Y:=\left(Y_{1}, Y_{2}, \ldots, Y_{l}\right)^{\operatorname{tr}} \in \mathbb{C}^{l} \backslash\{0\}$ such that

$\left|Y_{1} B_{i 1}+Y_{2} B_{i 2}+\cdots+Y_{l} B_{i l}\right|^{2}-\left|Y_{1} A_{i 1}+Y_{2} A_{i 2}+\cdots+Y_{l} A_{i l}\right|^{2}=0 \quad \forall i \in\{1,2,3,4,5\}$.

Let us recall that, for $p \in \mathbb{N} \backslash\{0\}, \mathcal{S}_{p}$ denotes the set of elements $Q \in \mathcal{M}_{p, p}$ such that $Q^{\operatorname{tr}}=Q$. For $i \in\{1,2,3,4,5\}$, there exists a unique $Q_{i} \in \mathcal{S}_{l}$ such that, for every $Y:=\left(Y_{1}, Y_{2}, \ldots, Y_{l}\right)^{\operatorname{tr}} \in \mathbb{C}^{l}$,

$$
Y^{\operatorname{tr}} Q_{i} \bar{Y}^{\operatorname{tr}}=\left|Y_{1} B_{i 1}+Y_{2} B_{i 2}+\cdots+Y_{l} B_{i l}\right|^{2}-\left|Y_{1} A_{i 1}+Y_{2} A_{i 2}+\cdots+Y_{l} A_{i l}\right|^{2},
$$

with $\bar{Y}:=\left(\bar{Y}_{1}, \bar{Y}_{2}, \ldots, \bar{Y}_{l}\right)(\bar{z}$ denoting the complex conjugate of $z \in \mathbb{C})$. Then (B.29) is equivalent to

$$
Y^{\operatorname{tr}} Q_{i} \bar{Y}^{\operatorname{tr}}=0 \quad \forall i \in\{1,2,3,4,5\} .
$$

For a matrix $M \in \mathcal{M}_{p, p}(\mathbb{C})$, let us denote by $\operatorname{tr}(M)$ its trace. Using (A.20) we have that

$$
\operatorname{tr}\left(Q_{i}\right)=0 \quad \forall i \in\{1,2,3,4,5\}
$$


Using (A.19), one gets that

$$
Y^{\operatorname{tr}} Q_{1} \bar{Y}^{\operatorname{tr}}+Y^{\operatorname{tr}} Q_{2} \bar{Y}+Y^{\operatorname{tr}} Q_{3} \bar{Y}+Y^{\operatorname{tr}} Q_{4} \bar{Y}+Y^{\operatorname{tr}} Q_{5} \bar{Y}=0 \quad \forall Y \in \mathbb{C}^{l} .
$$

Hence (B.30) is equivalent to

$$
Y^{\mathrm{tr}} Q_{i} \bar{Y}^{\mathrm{tr}}=0 \quad \forall i \in\{1,2,3,4\} .
$$

Therefore Proposition B.5 is a consequence of the following proposition due to Voisin [23].

Proposition B.6. Let $l \in\{3,4\}$. Let $Q_{1}, Q_{2}, Q_{3}$, and $Q_{4}$ be four elements of $\mathcal{S}_{l}$ such that

$$
\operatorname{tr}\left(Q_{i}\right)=0 \quad \forall i \in\{1,2,3,4\} .
$$

Then there exists $Y \in \mathbb{C}^{l} \backslash\{0\}$ such that

$$
Y^{\mathrm{tr}} Q_{i} \bar{Y}=0 \quad \forall i \in\{1,2,3,4\} .
$$

Proof of Proposition B.6. We reproduce the proof of [23]. For $l \in \mathbb{N}$, let $\overline{\mathcal{S}_{l,+}}$ be the set of semidefinite positive $S \in \mathcal{S}_{l}$. The first step is the following lemma.

Lemma B.7. Let $l, p$, and $n$ be three positive integers. Let $Q_{i}, i \in\{1, \ldots, n\}$, be $n$ elements of $\mathcal{S}_{l}$. Assume that

$$
\begin{gathered}
\operatorname{tr}\left(Q_{i}\right)=0 \quad \forall i \in\{1, \ldots, n\}, \\
n<\frac{(p+1)(p+2)}{2}-1 .
\end{gathered}
$$

Then there exists $S \in \overline{\mathcal{S}_{l,+}} \backslash\{0\}$ such that

the rank of $S$ is less than or equal to $p$,

$$
\operatorname{tr}\left(S Q_{i}\right)=0 \quad \forall i \in\{1, \ldots, n\} .
$$

Proof of Proposition B.7. Let

$$
C:=\left\{S \in \overline{\mathcal{S}_{l,+}} ; \operatorname{tr}(S)=l, \operatorname{tr}\left(S Q_{i}\right)=0 \quad \forall i \in\{1, \ldots, n\}\right\} .
$$

The set $C$ is a closed convex bounded subset of $\mathcal{M}_{l, l}(\mathbb{R})$. By (B.33), $\operatorname{Id}_{l} \in C$, and therefore $C$ is not empty. Hence, by the Krein-Milman theorem (see, e.g., [19, Theorem 3.21, page 70]), the convex set $C$ has at least an extreme point. Let $S$ be an extreme point of $C$. Then $S \in \overline{\mathcal{S}_{l,+}} \backslash\{0\}$ and satisfies (B.36). It remains only to check that (B.35) holds. Let $k$ be the rank of $S$. There exist an orthonormal matrix $O \in \mathcal{M}_{l, l}(\mathbb{R})$ and a definite positive matrix $S_{0} \in \mathcal{S}_{k}$ such that

$$
S=O^{\operatorname{tr}}\left(\begin{array}{cc}
S_{0} & 0 \\
0 & 0
\end{array}\right) O .
$$

Let

$$
\Pi:=\left\{O^{\operatorname{tr}}\left(\begin{array}{cc}
S^{\prime} & 0 \\
0 & 0
\end{array}\right) O ; S^{\prime} \in \mathcal{S}_{k}, \operatorname{tr}\left(S^{\prime}\right)=0\right\} \subset \mathcal{S}_{l} .
$$

Copyright $@$ by SIAM. Unauthorized reproduction of this article is prohibited. 
Let us assume that

$$
n<\frac{k(k+1)}{2}-1 \text {. }
$$

Since $\Pi$ is a vector subspace of $\mathcal{S}_{l}$ of dimension $(k(k+1) / 2)-1$, (B.39) implies that there exists $S_{0} \in \Pi \backslash\{0\}$ such that

$$
\operatorname{tr}\left(S_{0} Q_{i}\right)=0 \quad \forall i \in\{1, \ldots, n\}
$$

Then, for $\tau \in \mathbb{R}$ with $|\tau|$ small enough, $S+\tau S_{0}$ is in $C$, which contradicts the fact that $S$ is an extreme point of $C$. Hence (B.39) does not hold, which, together with (B.34), implies that $k \leqslant p$. This concludes the proof of Lemma B.7.

Let us go back to the proof of Proposition B.6. We apply Lemma B.7 with $n=4$ and $p=2$ (then (B.34) holds). We get the existence of $S \in \overline{\mathcal{S}_{l,+}} \backslash\{0\}$ satisfying

the rank of $\mathrm{S}$ is less than or equal to 2 ,

$$
\operatorname{tr}\left(S Q_{i}\right)=0 \quad \forall i \in\{1, \ldots, 4\}
$$

Let $\lambda_{1}>0, \lambda_{2} \geqslant 0$, and 0 be the eigenvalues of $S$. Let

$$
S_{0}=\left(\begin{array}{ccc}
\lambda_{1} & 0 & 0 \\
0 & \lambda_{2} & 0 \\
0 & 0 & 0
\end{array}\right) \in \overline{\mathcal{S}_{l,+}}
$$

There exists an orthonormal matrix $O$ such that

$$
S=O^{\operatorname{tr}} S_{0} O
$$

Let $Z:=\left(\sqrt{\lambda_{1}}, \iota \sqrt{\lambda_{2}}, 0\right) \in \mathbb{C}^{l} \backslash\{0\}$ and $Y:=O^{\operatorname{tr}} Z \in \mathbb{C}^{l} \backslash\{0\}$. Then, using (B.42) and (B.43), one gets that, for every $i \in\{1, \ldots, 4\}$,

$$
\begin{aligned}
2 Y^{\operatorname{tr}} Q_{i} \bar{Y}=\operatorname{tr}\left(\left(Y \bar{Y}^{\operatorname{tr}}+\bar{Y} Y\right)^{\operatorname{tr}} Q_{i}\right)=\operatorname{tr}\left(O^{\operatorname{tr}}\left(Z \bar{Z}^{\operatorname{tr}}+\bar{Z} Z^{\operatorname{tr}}\right) O Q_{i}\right) & \\
& =2 \operatorname{tr}\left(O^{\operatorname{tr}} S_{0}^{\operatorname{tr}} O Q_{i}\right)=\operatorname{tr}\left(S Q_{i}\right)=0
\end{aligned}
$$

which concludes the proof of Proposition B.6 and therefore the proof of Proposition B.5.

Finally, in order to end the proof of Proposition 3.7, it remains only to check that, for $n=6$ and therefore for every $n \geqslant 6$, there exists $K \in \mathcal{M}_{n, n}(\mathbb{R})$ such that $l=3$ and (3.24) hold. This is done in the following example.

Example B.8. Let $\left(u_{1}, v_{1}, w_{1}\right)^{\operatorname{tr}} \in \mathbb{R}^{3},\left(u_{2}, v_{2}, w_{2}\right)^{\operatorname{tr}} \in \mathbb{R}^{3},\left(x_{1}, y_{1}, z_{1}\right)^{\operatorname{tr}} \in \mathbb{R}^{3}$, and $\left(x_{2}, y_{2}, z_{2}\right)^{\text {tr }} \in \mathbb{R}^{3}$. We define $A_{1} \in \mathbb{R}^{6}, A_{2} \in \mathbb{R}^{6}, A_{3} \in \mathbb{R}^{6}, B_{1} \in \mathbb{R}^{6}, B_{2} \in \mathbb{R}^{6}$, and 
$B_{3} \in \mathbb{R}^{6}$ by

$$
\begin{gathered}
A_{1}:=\left(\begin{array}{c}
1 \\
0 \\
0 \\
1 \\
u_{1} \\
u_{2}
\end{array}\right), \quad A_{2}=\left(\begin{array}{c}
0 \\
1 \\
0 \\
0 \\
v_{1} \\
v_{2}
\end{array}\right), \quad A_{3}:=\left(\begin{array}{c}
0 \\
0 \\
1 \\
0 \\
w_{1} \\
w_{2}
\end{array}\right), \\
B_{1}:=\left(\begin{array}{c}
0 \\
0 \\
0 \\
1 / \sqrt{2} \\
x_{1} \\
x_{2}
\end{array}\right), \quad B_{2}:=\left(\begin{array}{c}
1 \\
0 \\
1 / \sqrt{2} \\
-1 / \sqrt{2} \\
y_{1} \\
y_{2}
\end{array}\right), \quad B_{3}:=\left(\begin{array}{c}
0 \\
1 \\
1 / \sqrt{2} \\
0 \\
z_{1} \\
z_{2}
\end{array}\right) .
\end{gathered}
$$

One easily checks that (A.20) holds if (and only if)

$$
\begin{aligned}
& u_{1}^{2}+v_{1}^{2}+w_{1}^{2}=x_{1}^{2}+y_{1}^{2}+z_{1}^{2}, \\
& u_{2}^{2}+v_{2}^{2}+w_{2}^{2}=x_{2}^{2}+y_{2}^{2}+z_{2}^{2} .
\end{aligned}
$$

Similarly (A.19) holds if (and only if)

$$
\begin{gathered}
\frac{3}{2}+u_{1}^{2}+u_{2}^{2}-x_{1}^{2}-x_{2}^{2}=0, \\
-1+v_{1}^{2}+v_{2}^{2}-y_{1}^{2}-y_{2}^{2}=0, \\
-\frac{1}{2}+w_{1}^{2}+w_{2}^{2}-z_{1}^{2}-z_{2}^{2}=0, \\
\frac{1}{2}+u_{1} v_{1}+u_{2} v_{2}-x_{1} y_{1}-x_{2} y_{2}=0, \\
u_{1} w_{1}+u_{2} w_{2}-x_{1} z_{1}-x_{2} z_{2}=0, \\
-\frac{1}{2}+v_{1} w_{1}+v_{2} w_{2}-y_{1} z_{1}-y_{2} z_{2}=0 .
\end{gathered}
$$

Note that (B.44), (B.46), (B.47), and (B.48) imply (B.45).

We take $l:=3$ and $R:=1$. We define $K \in \mathcal{M}_{6,6}(\mathbb{R})$ by requiring (A.21) and

$$
K X=0 \quad \forall X \in \mathbb{R}^{6} \text { such that } X^{\text {tr }} A_{1}=X^{\text {tr }} A_{2}=X^{\text {tr }} A_{3}=0 .
$$

From Proposition A.3 we get that if (B.44) and (B.46) to (B.51) hold, then

$$
\rho_{1}(K)=1 \text {. }
$$

Let us assume, for the moment, that (B.46) to (B.51) hold. If (3.24) does not hold, we have $\rho_{0}(K)=\rho_{1}(K)=1$, and therefore there exist $X \in \mathbb{C}^{6}$ and $\left(\Upsilon_{1}, \ldots, \Upsilon_{6}\right)^{\operatorname{tr}} \in \mathbb{C}^{n}$ such that (B.23), (B.24), and (B.25) hold. Clearly,

$$
|K X|=|X| \text {. }
$$


Since

$$
\begin{aligned}
|K(Y+Z)|=|Y| \quad \forall Y \in \mathbb{C} A_{1} & +\mathbb{C} A_{2}+\mathbb{C} A_{3}, \\
& \forall Z \in \mathbb{C}^{n} \text { such that } Z^{\operatorname{tr}} A_{1}=Z^{\operatorname{tr}} A_{2}=Z^{\operatorname{tr}} A_{3}=0,
\end{aligned}
$$

it follows from (B.52) that $X \in \mathbb{C} A_{1}+\mathbb{C} A_{2}+\mathbb{C} A_{3}$. Hence, there exist $\xi_{1} \in \mathbb{C}, \xi_{2} \in \mathbb{C}$, and $\xi_{3} \in \mathbb{C}$ such that

$$
X=\xi_{1} A_{1}+\xi_{2} A_{2}+\xi_{3} A_{3} .
$$

Using (B.25), one gets $(K X)_{1}=\Upsilon_{1} X_{1}$ and $(K X)_{2}=\Upsilon_{1} X_{2}$, which, together with (A.21) and (B.24), imply that

$$
\left|\xi_{1}\right|=\left|\xi_{2}\right|=\left|\xi_{3}\right| .
$$

Using (B.23) and (B.54) one sees that, without loss of generality, we may assume that

$$
\xi_{1}=1, \quad\left|\xi_{2}\right|=\left|\xi_{3}\right|=1 .
$$

Hence there exist $\theta_{2} \in \mathbb{R}$ and $\theta_{3} \in \mathbb{R}$ such that

$$
\xi_{2}=e^{i \theta_{2}}, \quad \xi_{3}=e^{i \theta_{3}} .
$$

Now using $\left|(K X)_{3}\right|=\left|X_{3}\right|$, one gets

$$
\left|\xi_{2}+\xi_{3}\right|=\sqrt{2}
$$

which, together with (B.55), is equivalent to

$$
\cos \left(\theta_{3}-\theta_{2}\right)=0
$$

i.e., there exists $\varepsilon_{3} \in\{1,-1\}$ such that

$$
\xi_{3}=\varepsilon_{3} \iota \xi_{2} .
$$

Proceeding similarly with the fourth of $K X$, one gets the existence of $\varepsilon_{2} \in\{1,-1\}$ such that

$$
\xi_{2}=\varepsilon_{2} \iota .
$$

Then $\left|(K X)_{5}\right|=\left|X_{5}\right|$ and $\left|(K X)_{6}\right|=\left|X_{6}\right|$ are equivalent to

$$
\begin{aligned}
& \left(u_{1}+\varepsilon_{1} w_{1}\right)^{2}+v_{1}^{2}=\left(x_{1}+\varepsilon_{1} z_{1}\right)^{2}+y_{1}^{2}, \\
& \left(u_{2}+\varepsilon_{1} w_{2}\right)^{2}+v_{2}^{2}=\left(x_{2}+\varepsilon_{1} z_{2}\right)^{2}+y_{2}^{2}
\end{aligned}
$$

with

$$
\varepsilon_{1}:=-\varepsilon_{2} \varepsilon_{3} \in\{1,-1\} .
$$

Let

$$
\begin{array}{cc}
F: \quad & \rightarrow \mathbb{R}^{7}, \\
P:=\left(u_{1}, v_{1}, w_{1}, x_{1}, y_{1}, z_{1}, u_{2}, v_{2}, w_{2}, x_{2}, y_{2}, z_{2}\right)^{\operatorname{tr}} & \mapsto F(P)
\end{array}
$$

Copyright $@$ ㅇ by SIAM. Unauthorized reproduction of this article is prohibited. 
be defined by

$$
F(P):=\left(\begin{array}{c}
\frac{3}{2}+u_{1}^{2}+u_{2}^{2}-x_{1}^{2}-x_{2}^{2} \\
-1+v_{1}^{2}+v_{2}^{2}-y_{1}^{2}-y_{2}^{2} \\
-\frac{1}{2}+w_{1}^{2}+w_{2}^{2}-z_{1}^{2}-z_{2}^{2} \\
\frac{1}{2}+u_{1} v_{1}+u_{2} v_{2}-x_{1} y_{1}-x_{2} y_{2} \\
u_{1} w_{1}+u_{2} w_{2}-x_{1} z_{1}-x_{2} z_{2} \\
-\frac{1}{2}+v_{1} w_{1}+v_{2} w_{2}-y_{1} z_{1}-y_{2} z_{2} \\
u_{1}^{2}+v_{1}^{2}+w_{1}^{2}-x_{1}^{2}-y_{1}^{2}-z_{1}^{2}
\end{array}\right)
$$

Let $\Sigma$ be the subset of $\mathbb{R}^{12}$ defined by

$$
\Sigma:=\left\{P \in \mathbb{R}^{12} ; F(P)=0 \text { and the rank of } F^{\prime}(P) \text { is } 7\right\} .
$$

Let

$$
\tilde{P}:=\left(0,1,0,1,0,0,-\frac{1}{4}, \frac{1}{2}, \frac{3}{4}, \frac{3}{4}, \frac{1}{2},-\frac{1}{4}\right)^{\mathrm{tr}} \in \mathbb{R}^{12}
$$

One easily checks that $F(\tilde{P})=0$. Straightforward computations give

$$
F^{\prime}(\tilde{P})=\left(\begin{array}{cccccccccccc}
0 & 0 & 0 & -2 & 0 & 0 & -\frac{1}{2} & 0 & 0 & -\frac{3}{2} & 0 & 0 \\
0 & 2 & 0 & 0 & 0 & 0 & 0 & 1 & 0 & 0 & -1 & 0 \\
0 & 0 & 0 & 0 & 0 & 0 & 0 & 0 & \frac{3}{2} & 0 & 0 & \frac{1}{2} \\
1 & 0 & 0 & 0 & -1 & 0 & \frac{1}{2} & -\frac{1}{4} & 0 & -\frac{1}{2} & -\frac{3}{4} & 0 \\
0 & 0 & 0 & 0 & 0 & -1 & \frac{3}{4} & 0 & -\frac{1}{4} & \frac{1}{4} & 0 & -\frac{3}{4} \\
0 & 0 & 1 & 0 & 0 & 0 & 0 & \frac{3}{4} & \frac{1}{2} & 0 & \frac{1}{4} & -\frac{1}{2} \\
0 & 2 & 0 & -2 & 0 & 0 & 0 & 0 & 0 & 0 & 0 & 0
\end{array}\right) .
$$

In particular, the rank of $F^{\prime}(\tilde{P})$ is 7 . Hence $\tilde{P}$ is in $\Sigma$, and the set $\Sigma$ is not empty and is a submanifold of $\mathbb{R}^{12}$ of dimension $12-7=5$. The tangent space to this manifold at $\tilde{P}$ is $\operatorname{Ker} F^{\prime}(\tilde{P})$. Let $G_{+}$be the map

$$
\begin{aligned}
& G_{+}: \quad \mathbb{R}^{12} \quad \rightarrow \quad \mathbb{R}^{2}, \\
& P:=\left(u_{1}, v_{1}, w_{1}, x_{1}, y_{1}, z_{1}, u_{2}, v_{2}, w_{2}, x_{2}, y_{2}, z_{2}\right)^{\operatorname{tr}} \mapsto G_{+}(P)
\end{aligned}
$$

defined by

$$
G_{+}(P):=\left(\begin{array}{l}
\left(u_{1}+w_{1}\right)^{2}+v_{1}^{2}-\left(x_{1}+z_{1}\right)^{2}-y_{1}^{2} \\
\left(u_{2}+w_{2}\right)^{2}+v_{2}^{2}-\left(x_{2}+z_{2}\right)^{2}-y_{2}^{2}
\end{array}\right)
$$

Copyright (C) by SIAM. Unauthorized reproduction of this article is prohibited. 
Similarly, let $G_{-}$be the map

$$
\begin{array}{ccc}
G_{-}: & \mathbb{R}^{12} & \rightarrow \mathbb{R}^{2}, \\
& P:=\left(u_{1}, v_{1}, w_{1}, x_{1}, y_{1}, z_{1}, u_{2}, v_{2}, w_{2}, x_{2}, y_{2}, z_{2}\right)^{\operatorname{tr}} & \mapsto G_{-}(P)
\end{array}
$$

defined by

$$
G_{-}(P):=\left(\begin{array}{l}
\left(u_{1}-w_{1}\right)^{2}+v_{1}^{2}-\left(x_{1}-z_{1}\right)^{2}-y_{1}^{2} \\
\left(u_{2}-w_{2}\right)^{2}+v_{2}^{2}-\left(x_{2}-z_{2}\right)^{2}-y_{2}^{2}
\end{array}\right) .
$$

Let $S_{+} \subset \mathbb{R}^{12}$ and $S_{-} \subset \mathbb{R}^{12}$ be defined by

$$
\begin{aligned}
& S_{+}:=\left\{P \in \mathbb{R}^{12} ; G_{+}(P)=0\right\}, \\
& S_{-}:=\left\{P \in \mathbb{R}^{12} ; G_{-}(P)=0\right\} .
\end{aligned}
$$

It suffices to check that

$$
\Sigma \text { is not a subset of } S_{-} \cup S_{+} \text {. }
$$

Note that $\tilde{P} \in S_{-} \cap S_{+}$and

$$
\begin{aligned}
G_{-}^{\prime}(\tilde{P}) & =\left(\begin{array}{cccccccccccc}
0 & 2 & 0 & -2 & 0 & 2 & 0 & 0 & 0 & 0 & 0 & 0 \\
0 & 0 & 0 & 0 & 0 & 0 & -2 & 1 & 2 & -2 & -1 & 2
\end{array}\right), \\
G_{+}^{\prime}(\tilde{P}) & =\left(\begin{array}{cccccccccccc}
0 & 2 & 0 & -2 & 0 & -2 & 0 & 0 & 0 & 0 & 0 & 0 \\
0 & 0 & 0 & 0 & 0 & 0 & 1 & 1 & 1 & -1 & -1 & -1
\end{array}\right) .
\end{aligned}
$$

In particular the rank of $G_{-}^{\prime}(\tilde{P})$ and the rank of $G_{+}^{\prime}(\tilde{P})$ are both equal to 2. Hence, if $r>0$ is small enough, the set $\left\{P \in S_{-} ;|P-\tilde{P}|<r\right\}$ and the set $\left\{P \in S_{+} ;|P-\tilde{P}|<r\right\}$ are submanifolds of $\mathbb{R}^{12}$ of dimension $12-2=10$ whose tangent spaces at $\tilde{P}$ are $\operatorname{Ker} G_{-}^{\prime}(\tilde{P})$ and $\operatorname{Ker} G_{+}^{\prime}(\tilde{P})$, respectively. Therefore (B.62) holds if

$$
\operatorname{Ker} F^{\prime}(\tilde{P}) \text { is not a subset of } \operatorname{Ker} G_{-}^{\prime}(\tilde{P}) \cup \operatorname{Ker} G_{+}^{\prime}(\tilde{P}) \text {. }
$$

Property (B.65) follows from (B.61), (B.63), and (B.64). This concludes the proof of Proposition 3.7.

\section{REFERENCES}

[1] A. Aw And M. Rascle, Resurrection of "second order" models of traffic flow, SIAM J. Appl. Math., 60 (2000), pp. 916-938.

[2] M. K. BAnda, M. Herty, And A. Klar, Gas flow in pipeline networks, Netw. Heterog. Media, 1 (2006), pp. 41-56.

[3] H. Brezis, Analyse fonctionnelle Théorie et applications, Collection Mathématiques Appliquées pour la Maîtrise, Masson, Paris, 1983.

[4] J.-M. Conon, On the null asymptotic stabilization of the two-dimensional incompressible Euler equations in a simply connected domain, SIAM J. Control Optim., 37 (1999), pp. 18741896.

[5] J.-M. Conon, Control and Nonlinearity, Math. Surveys Monogr. 136, AMS, Providence, RI, 2007.

Copyright (C) by SIAM. Unauthorized reproduction of this article is prohibited. 
[6] J.-M. Coron, B. D'Andréa-Novel, And G. Bastin, A strict Lyapunov function for boundary control of hyperbolic systems of conservation laws, IEEE Trans. Automat. Control, 52 (2007), pp. 2-11.

[7] J. de Halleux, C. Prieur, J.-M. Coron, B. D'Andréa-Novel, and G. Bastin, Boundary feedback control in networks of open channels, Automatica J. IFAC, 39 (2003), pp. 13651376.

[8] M. Garavello and B. Piccoli, Traffic flow on a road network using the Aw-Rascle model, Comm. Partial Differential Equations, 31 (2006), pp. 243-275.

[9] J. M. Greenberg and T. T. LI, The effect of boundary damping for the quasilinear wave equation, J. Differential Equations, 52 (1984), pp. 66-75.

[10] J. K. Hale And S. M. Verduyn Lunel, Introduction to Functional-Differential Equations, Appl. Math. Sci. 99, Springer-Verlag, New York, 1993.

[11] J. K. Hale And S. M. Verduyn Lunel, Strong stabilization of neutral functional differential equations, IMA J. Math. Control Inform., 19 (2002), pp. 5-23.

[12] T. Kato, The Cauchy problem for quasi-linear symmetric hyperbolic systems, Arch. Rational Mech. Anal., 58 (1975), pp. 181-205.

[13] P. D. LAx, Hyperbolic Systems of Conservation Laws and the Mathematical Theory of Shock Waves, CBMS-NSF Regional Conf. Ser. Appl. Math. 11, SIAM, Philadelphia, 1973.

[14] T. T. LI, Global Classical Solutions for Quasilinear Hyperbolic Systems, RAM Res. Appl. Math. 32, Masson, Paris, 1994.

[15] T. T. Li And W. C. YU, Boundary Value Problems for Quasilinear Hyperbolic Systems, Duke Univ. Math. Ser. V, Duke University Mathematics Department, Durham, NC, 1985.

[16] A. MajDA, Compressible Fluid Flow and Systems of Conservation Laws in Several Space Variables, Appl. Math. Sci. 53, Springer-Verlag, New York, 1984.

[17] T. H. QIN, Global smooth solutions of dissipative boundary value problems for first order quasilinear hyperbolic systems, Chinese Ann. Math. Ser. B, 6 (1985), pp. 289-298; a Chinese summary appears in Chinese Ann. Math. Ser. A, 6 (1985), p. 514.

[18] M. Reed And B. Simon, Methods of Modern Mathematical Physics. IV. Analysis of Operators, Academic Press, New York, 1978.

[19] W. Rudin, Functional Analysis, McGraw-Hill Series in Higher Mathematics, McGraw-Hill, New York, 1973.

[20] D. Serre, Systèmes de Lois de Conservation. I., Hyperbolicité, Entropies, Ondes de Choc, Fondations, Diderot Editeur, Paris, 1996.

[21] M. Slemrod, Boundary feedback stabilization for a quasilinear wave equation, in Control Theory for Distributed Parameter Systems and Applications, Lecture Notes in Control and Inform. Sci. 54, Springer-Verlag, Berlin, 1983, pp. 221-237.

[22] A. TChousso, T. Besson, And C.-Z. Xu, Exponential stability of distributed parameter systems governed by symmetric hyperbolic partial differential equations using Lyapunov's second method, ESAIM Control Optim. Calc. Var., to appear.

[23] C. VoIsin, private communication, 2007.

[24] C.-Z. XU AND G. SALlet, Exponential stability and transfer functions of processes governed by symmetric hyperbolic systems, ESAIM Control Optim. Calc. Var., 7 (2002), pp. 421-442.

[25] Y. C. ZнаO, The boundary value problem for systems of first-order quasilinear hyperbolic equations, Chinese Ann. Math. Ser. A, 7 (1986), pp. 629-643; an English summary appears in Chinese Ann. Math. Ser. B, 8 (1987), pp. 127-128.

[26] L. A. ŽivotovskiI , Absolute stability of the solutions of differential equations with several lags, Trudy Sem. Teor. Differencial. Uravneniı̌ s Otklon. Argumentom Univ. Družby Narodov Patrisa Lumumby, 7 (1969), pp. 82-91.

Copyright $@$ by SIAM. Unauthorized reproduction of this article is prohibited. 